\title{
QUANTUM CORRESPONDENCES OF AFFINE LIE SUPERALGEBRAS
}

\author{
YING XU AND R. B. ZHANG
}

\begin{abstract}
There is a surprising isomorphism between the quantised universal enveloping algebras of $\mathfrak{p s p}(1 \mid 2 n)$ and $\mathfrak{s p}(2 n+1)$. This same isomorphism emerged in recent work of Mikhaylov and Witten in the context of string theory as a $T$-duality composed with an S-duality. We construct similar Hopf superalgebra isomorphisms for families of pairs of quantum affine superalgebras. An immediate consequence is that the representation categories of the quantum affine superalgebras in each pair are equivalent as strict tensor categories.
\end{abstract}

\section{INTRODUCTION}

In the early 80s, Rittenberg and Scheunert [12] observed a remarkable connection between the orthogonal Lie algebra $\mathfrak{s} \mathfrak{0}(2 n+1)$ and the orthosymplectic Lie superalgebra $\mathfrak{D} \mathfrak{s p}(1 \mid 2 n)$ : there is a one-to-one correspondence between the finite dimensional representations of $\mathfrak{b s p}(1 \mid 2 n)$ and the tensorial representations of $\mathfrak{s} \mathfrak{D}(2 n+1)$, and the central characters of the two algebras in the corresponding simple modules are the same. This remained a mystery until quantum supergroups [2, 15, 22, 18, 20] came to the scene. It was discovered in [17] that the quantised universal enveloping algebras of these Lie (super)algebras are essentially isomorphic, and the Rittenberg-Scheunert correspondence is a consequence of this isomorphism in the semiclassical limits.

The isomorphism between the quantum (super)groups of $\mathfrak{D s p}(1 \mid 2 n)$ and $\mathfrak{s} \mathfrak{p}(2 n+1)$ was used by Lanzmann to great effect [8] in the study of primitive ideals of $\mathrm{U}(\mathfrak{D} \mathfrak{s p}(1 \mid 2 n))$. By relating them to the primitive ideals of $\mathrm{U}(\mathfrak{s v}(2 n+1))$ via the isomorphism, he drastically simplified the proofs of the results first obtained in [4].

The same isomorphism emerged in very recent work of Mikhaylov and Witten [10] on quantum Chern-Simons theories. The authors gave a description of Chern-Simons theories with super gauge groups in terms of systems of D3-branes ending on 2-sides of an NS5-brane. A T-duality composed with an S-duality of the brane systems interchanges the corresponding quantum Chern-Simons theories with gauge groups $\mathfrak{b s p}(1 \mid 2 n)$ and $\mathfrak{s o}(2 n+1)$ respectively. The strong-weak coupling transformation procured by the T-duality corresponds precisely to the interchange $q \leftrightarrow-q$ in the quantum group context [17]. Furthermore, a similar duality between quantum Chern-Simons theories with gauge groups $\mathfrak{D s p}(2 m+1 \mid 2 n)$ and $\mathfrak{D} \mathfrak{s p}(2 n+1 \mid 2 m)$ was constructed for arbitrary $m$ and $n$ in [10].

The aim of the present paper is to give a catalogue of the isomorphisms analogous to that between the quantum (super)groups of $\mathfrak{p} \mathfrak{s p}(1 \mid 2 n)$ and $\mathfrak{s} \mathfrak{v}(2 n+1)$. The main results are summarised in Theorem 1.2. To explain the content of the theorem, we need several conceptual constructs.

2010 Mathematics Subject Classification. 15A72,17B20.

Key words and phrases. affine Lie superalgebras, quantum affine superalgebras, Hopf superalgebras, tensor categories. 
Let $\mathfrak{g}$ be a Lie superalgebra or an affine Lie superalgebra in the classical series. Fixing a fundamental system $\Pi$ of simple roots (Definition 2.4) corresponding to an arbitrary choice of Borel subalgebra, we let $\mathrm{U}_{q}(\mathfrak{g}, \Pi)$ be the quantised universal enveloping superalgebra of $\mathfrak{g}$ with respect to this fundamental system. We note that in general, the quantised universal enveloping superalgebras $\mathrm{U}_{q}(\mathfrak{g}, \Pi)$ associated with different fundamental systems $\Pi$ are not isomorphic as Hopf superalgebras.

Corresponding to each $\alpha_{i}$ in $\Pi$, we introduce a $\mathbb{Z}_{2}$ group generated by $\sigma_{i}$ such that $\sigma_{i}^{2}=1$, and let $G$ be the direct product of all the $\mathbb{Z}_{2}$ groups, i.e., $G:=\mathbb{Z}_{2} \times \cdots \times \mathbb{Z}_{2}(|\Pi|$ copies). There is a natural action of $\mathrm{G}$ on $\mathrm{U}_{q}(\mathfrak{g}, \Pi)$ such that there exists an element $u \in \mathrm{G}$ which implements the $\mathbb{Z}_{2}$-grading. Let $\mathfrak{U}_{q}(\mathfrak{g}, \Pi)=\mathrm{U}_{q}(\mathfrak{g}, \Pi) \sharp \mathbb{C} G$ be the smash product (Definition 2.6), which has a natural Hopf superalgebra structure by Proposition 2.7.

The following result is a part of Theorem 1.2 .

Theorem 1.1. Let $\left(\mathfrak{g}, \mathfrak{g}^{\prime}\right)$ be a pair of Lie superalgebras or affine Lie superalgebras listed in any column of Table 1 (where $m+n>0)$.

TABLE 1. Quantum correspondences

\begin{tabular}{r|c|c|c}
\hline $\mathfrak{g}$ & $\mathfrak{D} \mathfrak{s p}(2 m+1 \mid 2 n)$ & $\mathfrak{s l}(2 m+1 \mid 2 n)^{(2)}$ & $\mathfrak{D} \mathfrak{s p}(2 m+2 \mid 2 n)^{(2)}$ \\
\hline $\mathfrak{g}^{\prime}$ & $\mathfrak{D} \mathfrak{s p}(2 n+1 \mid 2 m)$ & $\mathfrak{D} \mathfrak{p}(2 n+1 \mid 2 m)^{(1)}$ & $\mathfrak{D} \mathfrak{s p}(2 n+2 \mid 2 m)^{(2)}$ \\
\hline
\end{tabular}

For any chosen fundamental system $\Pi$ of $\mathfrak{g}$ and the corresponding fundamental system $\Pi^{\prime}=\phi(\Pi)$ of $\mathfrak{g}^{\prime}$ (see Lemma 2.2), there exists an isomorphism of associative algebras

$$
\mathfrak{U}_{-q}\left(\mathfrak{g}^{\prime}, \Pi^{\prime}\right) \stackrel{\cong}{\longrightarrow} \mathfrak{U}_{q}(\mathfrak{g}, \Pi),
$$

which is defined explicitly in Theorem 3.1

For the special pairs $\left(\mathfrak{g}, \mathfrak{g}^{\prime}\right)$ with either $\mathfrak{g}$ or $\mathfrak{g}^{\prime}$ being an ordinary affine Lie algebra, Theorem 1.1 was proved in [17, 19]. It was quite a surprise that such isomorphisms exist as the root systems of any pair $\mathfrak{g}$ and $\mathfrak{g}^{\prime}$ are very different.

As it stands, the isomorphism in Theorem 1.1 does not preserve the $\mathbb{Z}_{2}$-gradings, thus can not be a superalgebra isomorphism, let alone a Hopf superalgebra isomorphism. However, it relates the Hopf superalgebra structures at a more fundamental level.

As advocated by Majid and others (see e.g., [9, Chapter 10.1] and [1]), one should place Hopf superalgebras in the context of braided tensor categories. The category of vector superspaces can be considered as the tensor category of representations of the group algebra of $\mathbb{Z}_{2}$ regarded as a triangular Hopf algebra. A Hopf superalgebra is then a Hopf algebra in this category. Given a Hopf superalgebra, one may change the $\mathbb{Z}_{2}$-action to obtain a new Hopf superalgebra with the same underlying associative algebra structure, but a new co-algebra structure and different $\mathbb{Z}_{2}$-grading. We refer to this process as a picture change (see Definition 4.1), which is also loosely known as bosonisation in the literature [9] (see Remark 4.2). An important fact is that the representation category of the new Hopf superalgebra is equivalent to that of the original Hopf superalgebra as strict tensor category (see [9, Chapter 10.1] and [1, Theorem 3.1.1]).

Corresponding to any pair $\left(\mathfrak{g}, \mathfrak{g}^{\prime}\right)$ of (affine) Lie superalgebras from Theorem 1.1, we have the Hopf superalgebras $\mathfrak{U}_{q}(\mathfrak{g}, \Pi)$ and $\mathfrak{U}_{-q}\left(\mathfrak{g}^{\prime}, \Pi^{\prime}\right)$ with the standard Hopf structures. Denote by $\Delta, \epsilon$ and $S$ the co-multiplication, co-unit and antipode of $\mathfrak{U}_{q}(\mathfrak{g}, \Pi)$ respectively. We apply an appropriate picture change to $\left(\mathfrak{U}_{q}(\mathfrak{g}, \Pi), \Delta, \epsilon, S\right)$ to obtain a new Hopf superalgebra $\left(\mathfrak{U}_{q}(\mathfrak{g}, \Pi), \tilde{\Delta}, \epsilon, \tilde{S}\right)$, where $\mathfrak{U}_{q}(\mathfrak{g}, \Pi)$ has acquired a new $\mathbb{Z}_{2}$-grading. Relative to 
this $\mathbb{Z}_{2}$-grading, the map of Theorem 1.1 becomes an isomorphism of superalgebras; see Corollary 4.5 .

However, $\mathfrak{U}_{-q}\left(\mathfrak{g}^{\prime}, \Pi^{\prime}\right)$ and $\left(\mathfrak{U}_{q}(\mathfrak{g}, \Pi), \tilde{\Delta}, \epsilon, \tilde{S}\right)$ as Hopf superalgebras are still different. To relate them, we introduce another ingredient, Drinfeld twists [3, 11], which is used for changing the co-algebraic structures. We construct a Drinfeld twist $\mathcal{J}$ for $\left(\mathfrak{U}_{q}(\mathfrak{g}, \Pi), \tilde{\Delta}, \epsilon, \tilde{S}\right)$ in Lemma 4.6, and use it to twist the Hopf superalgebra in the way described in Section 4.1.2. This gives rise to another Hopf superalgebra $\left(\mathfrak{U}_{q}(\mathfrak{g}, \Pi), \tilde{\Delta}^{\mathcal{J}}, \epsilon, \tilde{S}^{\mathcal{J}}\right)$, see Lemma 4.8.

Theorem 1.2. Let $\left(\mathfrak{g}, \mathfrak{g}^{\prime}\right)$ be a pair of (affine) Lie superalgebras in Theorem 1.1 Then the quantum (affine) superalgebra $\mathfrak{U}_{-q}\left(\mathfrak{g}^{\prime}, \Pi^{\prime}\right)$ with the standard Hopf superalgebra structure is isomorphic to $\left(\mathfrak{U}_{q}(\mathfrak{g}, \Pi), \tilde{\Delta}^{\mathcal{J}}, \epsilon, \tilde{S}^{\mathcal{J}}\right)$.

We comment that even though Theorem 1.1 was partially known [17, 19] before, the Hopf superalgebra isomorphism of Theorem 1.2 is new in all cases. In general, the quantised universal enveloping superalgebras $\mathrm{U}_{\mathrm{q}}(\mathfrak{g}, \Pi)$ corresponding to different fundamental systems are non-isomorphic as Hopf superalgebras, thus the isomorphism of Theorem 1.2 depends on the fundamental systems nontrivially.

Definition 1.3. We call the Hopf superalgebra isomorphism of Theorem 1.2 a quantum correspondence between the (affine) Lie superalgebras $\mathfrak{g}$ and $\mathfrak{g}^{\prime}$.

The following result is a consequence of the quantum correspondence and some general facts (see Theorem 4.3) on Hopf superalgebras.

Theorem 1.4. Let $\left(\mathfrak{g}, \mathfrak{g}^{\prime}\right)$ be any pair of (affine) Lie superalgebras in Theorem 1.1 For any fundamental system $\Pi$ of $\mathfrak{g}$ and the corresponding fundamental system $\Pi^{\prime}=\phi(\Pi)$ of $\mathfrak{g}^{\prime}$, the representation categories of the Hopf superalgebras $\mathfrak{U}_{q}(\mathfrak{g}, \Pi)$ and $\mathfrak{U}_{-q}\left(\mathfrak{g}^{\prime}, \Pi^{\prime}\right)$ are equivalent as strict tensor categories.

The remainder of the paper is devoted to the proof of Theorem 1.2. All notions required, including those used in the discussion above, will be carefully explained.

Results of the present paper have been applied to construct Drinfeld realisations [14], vertex operator representations, and finite dimensional representations of classes of quantum affine superalgebras.

\section{QUANTISED UNIVERSAL ENVELOPING SUPERALGEBRAS}

2.1. Root Systems. We begin with a description of the root data of the classical series of Lie superalgebras and the related twisted and untwisted affine Lie superalgebras.

For any given pair of nonnegative integers $k$ and $l$, we let $\mathcal{E}(k \mid l)$ be the $(k+l)$-dimensional vector space over $\mathbb{R}$ with a basis consisting of elements $\varepsilon_{i}(i=1,2, \ldots, k)$ and $\delta_{v}(v=$ $1,2, \ldots, l)$. We endow $\mathcal{E}(k \mid l)$ with a symmetric non-degenerate bi-linear form

$$
\left(\varepsilon_{i}, \varepsilon_{j}\right)=(-1)^{\theta} \delta_{i j}, \quad\left(\delta_{\mu}, \delta_{v}\right)=-(-1)^{\theta} \delta_{\mu \nu}, \quad\left(\varepsilon_{i}, \delta_{\mu}\right)=\left(\delta_{\mu}, \varepsilon_{i}\right)=0,
$$

where $\theta$ is 0 or 1 which will be fixed in the following way. Call an order of the basis elements admissible if $\varepsilon_{i}$ appears before $\varepsilon_{i+1}$ for all $i$, and $\delta_{v}$ before $\delta_{v+1}$ for all $v$. Fix an admissible order and denote by $\left(\mathcal{E}_{1}, \mathcal{E}_{2}, \ldots, \mathcal{E}_{k+l}\right)$ the ordered basis of $\mathcal{E}(k \mid l)$. Then we choose $\theta$ so that $\left(\mathcal{E}_{1}, \mathcal{E}_{1}\right)=1$. 
Let $\mathfrak{g}$ be either a special linear or orthosymplectic Lie superalgebra. Then the set $\Phi$ of roots of $\mathfrak{g}$ can be realized as a subset of $\mathcal{E}(k \mid l)$ for appropriate $k$ and $l$, where we will take the $k$ and $l$ to be the smallest possible. We will call $\mathcal{E}(k \mid l)$ the ambient space of $\Phi$.

Each choice of a Borel subalgebra corresponds to a choice of positive roots, and hence a fundamental system $\Pi=\left\{\alpha_{1}, \alpha_{2}, \ldots, \alpha_{r}\right\}$ of simple roots, where $r$ is the rank of $\mathfrak{g}$. The Weyl group conjugacy classes of Borel subalgebras correspond bijectively to the admissible ordered bases of the ambient space.

The root data of the classical series of simple Lie superalgebras can be described as in Table 2, where the ambient space of $\Phi$ is $\mathcal{E}(m \mid n)$ in each case. Now $\Phi \subset \mathcal{E}(m \mid n)_{\mathbb{Z}}=$ $\sum_{a=1}^{m+n} \mathbb{Z} \mathcal{E}_{a}$. Define a map $\left.\chi: \mathcal{E}(m \mid n)\right)_{\mathbb{Z}} \longrightarrow \mathbb{Z}$ such that $\chi(v)=\sum_{\mu=1}^{n} b_{\mu}$ for any $v=$ $\sum_{i=1}^{m} a_{i} \varepsilon_{i}+\sum_{\mu=1}^{n} b_{\mu} \delta_{\mu}$. Then a root $\beta \in \Phi$ is even if $\chi(\beta)$ is even, and odd otherwise.

TABLE 2. Classical series of Lie superalgebras

\begin{tabular}{c|c}
\hline $\mathfrak{g}$ & simple roots \\
\hline $\mathfrak{s l}(m \mid n)$ & $\alpha_{i}=\mathcal{E}_{i}-\mathcal{E}_{i+1}, \quad 1 \leq i<m+n$ \\
\hline $\mathfrak{D} \mathfrak{s p}(2 m+1 \mid 2 n)$ & $\alpha_{i}=\mathcal{E}_{i}-\mathcal{E}_{i+1}, 1 \leq i<m+n, \quad \alpha_{m+n}=\mathcal{E}_{m+n}$ \\
\hline $\mathfrak{D} \mathfrak{s p}(2 m \mid 2 n)$ & $\alpha_{i}=\mathcal{E}_{i}-\mathcal{E}_{i+1}, 1 \leq i<m+n$, \\
& $\alpha_{m+n}= \begin{cases}\mathcal{E}_{m+n-1}+\mathcal{E}_{m+n}, & \text { if } \mathcal{E}_{m+n}=\varepsilon_{m}, \\
2 \mathcal{E}_{m+n}, & \text { if } \mathcal{E}_{m+n}=\delta_{n} . \\
\hline\end{cases}$
\end{tabular}

Remark 2.1. The Lie superalgebras $\mathfrak{D} \mathfrak{s p}(m \mid n)$ and $\mathfrak{s l}(m \mid n)$ reduce to ordinary Lie algebras if $m=0$ or $n=0$. Also, $\mathfrak{s l}(m \mid m)$ contains the ideal $\mathbb{C} 1_{2 m}$, and $\mathfrak{s l}(m \mid m) / \mathbb{C} 1_{2 m}$ is simple.

In order to describe the root data of untwisted and twisted affine Lie superalgebras of the classical series of Lie superalgebras discussed above, we introduce the vector space $\mathcal{E}_{\delta}(k \mid l)$, which has a basis consisting of the basis elements of $\mathcal{E}(k \mid l)$ and the additional element $\mathcal{E}_{0}=\delta$. We extend the bilinear form on $\mathcal{E}(k \mid l)$ to $\mathcal{E}_{\delta}(k \mid l)$ by setting

$$
\left(\mathcal{E}_{0}, \mathcal{E}_{i}\right)=\left(\mathcal{E}_{i}, \mathcal{E}_{0}\right)=0, \forall i=0,1, \ldots, k+l .
$$

The resulting form still has rank $k+l$ and is degenerate. The affine root data can be described as in Table 3 (see [6, 7, 13]) using the ambient space $\mathcal{E}_{\delta}(m \mid n)$ in each case.

The vector spaces $\mathcal{E}_{\delta}(m \mid n)$ and $\mathcal{E}_{\delta}(n \mid m)$ are both $(m+n+1)$-dimensional. To avoid confusion, we write the basis of $\mathcal{E}_{\delta}(n \mid m)$ as $\left\{\delta^{\prime}, \varepsilon_{1}^{\prime}, \ldots \varepsilon_{n}^{\prime}, \delta_{1}^{\prime}, \ldots \delta_{m}^{\prime}\right\}$. Consider the following vector space isomorphism

$$
\phi: \mathcal{E}_{\delta}(m \mid n) \longrightarrow \mathcal{E}_{\delta}(n \mid m), \quad \delta \mapsto \delta^{\prime}, \quad \varepsilon_{i} \mapsto \delta_{i}^{\prime}, \quad \delta_{j} \mapsto \varepsilon_{j}^{\prime}, \quad \forall i, j
$$

We will still denote its restriction to $\mathcal{E}(m \mid n)$ by $\phi$.

Clearly $\phi$ sends an admissible basis of $\mathcal{E}(m \mid n)$ to an admissible basis of $\mathcal{E}(n \mid m)$. If $\mathcal{E}(m \mid n)$ or $\mathcal{E}_{\delta}(m \mid n)$ is the ambient space of the root system of a Lie superalgebra or affine Lie superalgebra $\mathfrak{g}$, and $\Pi$ is a fundamental system of $\mathfrak{g}$, then the set $\phi(\Pi)$ may be a fundamental system of another (affine) Lie superalgebra with $\mathcal{E}(n \mid m)$ or $\mathcal{E}_{\delta}(n \mid m)$ as the ambient space of roots. This happens in the following cases.

Lemma 2.2. The map $\phi$ induces a one to one correspondences between fundamental systems of the (affine) Lie superalgebras in each of the following pairs $\left(\mathfrak{g}, \mathfrak{g}^{\prime}\right)$ :

(i). those listed in Table 1;

(ii). and $(\mathfrak{s l}(m \mid n), \mathfrak{s l}(n \mid m)),\left(\mathfrak{s l}(m \mid n)^{(1)}, \mathfrak{s l}(n \mid m)^{(1)}\right),\left(\mathfrak{s l}(2 m \mid 2 n)^{(2)}, \mathfrak{s l}(2 n \mid 2 m)^{(2)}\right)$, $\left(\mathfrak{s l}(2 m+1 \mid 2 n)^{(2)}, \mathfrak{s l}(2 n \mid 2 m+1)^{(2)}\right),\left(\mathfrak{s l}(2 m+1 \mid 2 n+1)^{(4)}, \mathfrak{s l}(2 n+1 \mid 2 m+1)^{(4)}\right)$. 
QUANTUM CORRESPONDENCES

TABLE 3. Classical series of affine Lie superalgebras

\begin{tabular}{|c|c|}
\hline $\mathfrak{g}$ & simple roots \\
\hline $\mathfrak{s l}(m \mid n)^{(1)}$ & $\begin{array}{c}\alpha_{i}=\mathcal{E}_{i}-\mathcal{E}_{i+1}, \quad 1 \leq i<m+n \\
\alpha_{0}=\mathcal{E}_{0}-\mathcal{E}_{1}+\mathcal{E}_{m+n}\end{array}$ \\
\hline $\mathfrak{D} \mathfrak{s p}(2 m+1 \mid 2 n)^{(1)}$ & $\begin{array}{c}\alpha_{i}=\mathcal{E}_{i}-\mathcal{E}_{i+1}, \quad 1 \leq i<m+n, \quad \alpha_{m+n}=\mathcal{E}_{m+n}, \\
\alpha_{0}= \begin{cases}\mathcal{E}_{0}-\mathcal{E}_{1}-\mathcal{E}_{2}, & \text { if } \mathcal{E}_{1}=\varepsilon_{1}, \\
\mathcal{E}_{0}-2 \mathcal{E}_{1}, & \text { if } \mathcal{E}_{1}=\delta_{1} .\end{cases} \end{array}$ \\
\hline $\mathfrak{D} \mathfrak{s p}(2 m \mid 2 n)^{(1)}$ & $\begin{array}{c}\alpha_{i}=\mathcal{E}_{i}-\mathcal{E}_{i+1}, \quad 1 \leq i<m+n, \\
\alpha_{m+n}= \begin{cases}\mathcal{E}_{m+n-1}+\mathcal{E}_{m+n}, & \text { if } \mathcal{E}_{m+n}=\varepsilon_{m}, \\
2 \mathcal{E}_{m+n}, & \text { if } \mathcal{E}_{m+n}=\delta_{n},\end{cases} \\
\alpha_{0}= \begin{cases}\mathcal{E}_{0}-\mathcal{E}_{1}-\mathcal{E}_{2}, & \text { if } \mathcal{E}_{1}=\varepsilon_{1}, \\
\mathcal{E}_{0}-2 \mathcal{E}_{1}, & \text { if } \mathcal{E}_{1}=\delta_{1} .\end{cases} \end{array}$ \\
\hline $\mathfrak{s l}(2 m+1 \mid 2 n)^{(2)}$ & $\begin{array}{c}\alpha_{i}=\mathcal{E}_{i}-\mathcal{E}_{i+1}, \quad 1 \leq i<m+n, \quad \alpha_{m+n}=\mathcal{E}_{m+n}, \\
\alpha_{0}= \begin{cases}\mathcal{E}_{0}-2 \mathcal{E}_{1}, & \text { if } \mathcal{E}_{1}=\varepsilon_{1}, \\
\mathcal{E}_{0}-\mathcal{E}_{1}-\mathcal{E}_{2}, & \text { if } \mathcal{E}_{1}=\delta_{1}\end{cases} \end{array}$ \\
\hline $\mathfrak{s l}(2 m \mid 2 n)^{(2)}$ & $\begin{array}{c}\alpha_{i}=\mathcal{E}_{i}-\mathcal{E}_{i+1} 1 \leq i<m+n, \\
\alpha_{m+n}= \begin{cases}\mathcal{E}_{m+n-1}+\mathcal{E}_{m+n}, & \text { if } \mathcal{E}_{m+n}=\varepsilon_{m}, \\
2 \mathcal{E}_{m+n}, & \text { if } \mathcal{E}_{m+n}=\delta_{n},\end{cases} \\
\alpha_{0}= \begin{cases}\mathcal{E}_{0}-2 \mathcal{E}_{1}, & \text { if } \mathcal{E}_{1}=\varepsilon_{1}, \\
\mathcal{E}_{0}-\mathcal{E}_{1}-\mathcal{E}_{2}, & \text { if } \mathcal{E}_{1}=\delta_{1} .\end{cases} \end{array}$ \\
\hline $\mathfrak{D} \mathfrak{s p}(2 m+2 \mid 2 n)^{(2)}$ & $\begin{array}{l}\alpha_{i}=\mathcal{E}_{i}-\mathcal{E}_{i+i} \quad 1 \leq i<m+n \\
\alpha_{m+n}=\mathcal{E}_{m+n}, \quad \alpha_{0}=\mathcal{E}_{0}-\mathcal{E}_{1}\end{array}$ \\
\hline $\mathfrak{s l}(2 m+1 \mid 2 n+1)^{(4)}$ & $\begin{array}{l}\alpha_{i}=\mathcal{E}_{i}-\mathcal{E}_{i+i} \quad 1 \leq i<m+n \\
\alpha_{m+n}=\mathcal{E}_{m+n}, \quad \alpha_{0}=\mathcal{E}_{0}-\mathcal{E}_{1}\end{array}$ \\
\hline
\end{tabular}

Remark 2.3. (1). The imaginary root $\mathcal{E}_{0}=\delta$ is even for all affine Lie superalgebras except $\mathfrak{s l}(2 m+1 \mid 2 n+1)^{(4)}$, where it is odd.

(2). Define a map $\chi_{\mathfrak{g}}: \mathbb{Z} \delta+\mathcal{E}(m \mid n)_{\mathbb{Z}} \longrightarrow \mathbb{Z}$ for each $\mathfrak{g}$ in Table 3 as follows. For any $v=z_{0} \delta+v^{\prime}$ with $v^{\prime} \in \mathcal{E}(m \mid n)_{\mathbb{Z}}$, let $\chi_{\mathfrak{g}}(v)=z_{0}+\chi\left(v^{\prime}\right)$ if $\mathfrak{g}=\mathfrak{s t}(2 m+1 \mid 2 n+1)^{(4)}$, and $\chi_{\mathfrak{g}}(v)=\chi\left(v^{\prime}\right)$ otherwise. Then a simple root $\alpha_{i}$ is even if $\chi_{\mathfrak{g}}\left(\alpha_{i}\right)$ is even, and odd otherwise. (3). For all the pairs in case (ii) of Lemma 2.2, we have $\mathfrak{g}=\mathfrak{g}^{\prime}$. This is why we do not consider them when studying quantum correspondences.

2.2. Quantum affine superalgebras. Hereafter we will only consider the Lie superalgebras in Table 2 and affine Lie superalgebras in Table 3 .

Let $\mathfrak{g}$ be such a Lie superalgebra or affine Lie superalgebra with a fundamental system $\Pi$. For $\mathfrak{g}$ in Table 2, let $\Pi=\left\{\alpha_{i} \mid i=1,2, \ldots, m+n\right\}$, and let $\tau \subset\{1,2, \ldots, m+n\}$ be the labelling set of the odd simple roots, i.e., $\left\{\alpha_{s} \mid s \in \tau\right\}$ is the subset of $\Pi$ consisting of the odd simple roots. Similarly, for $\mathfrak{g}$ in Table 3 , let $\Pi=\left\{\alpha_{i} \mid i=0,1,2, \ldots, m+n\right\}$, and let $\tau \subset\{0,1,2, \ldots, m+n\}$ be the labelling set of the odd simple roots. Define $b_{i j}=\left(\alpha_{i}, \alpha_{j}\right)$ 
for all $i, j$. Then the Cartan matrix of $\mathfrak{g}$ corresponding to $\Pi$ is given by

$$
A=\left(a_{i j}\right) \quad \text { with } \quad a_{i j}=\left\{\begin{array}{ll}
\frac{2 b_{i j}}{b_{i i}}, & \text { if } b_{i i} \neq 0 \\
b_{i j}, & \text { if } b_{i i}=0
\end{array} .\right.
$$

Note that $a_{i i}=0$ if and only if $\alpha_{i}$ is an isotropic odd simple root. We will represent fundamental systems by Dynkin diagrams [6, 7, 13, 21], following the convention of Kac [6]. In particular, a node $\circ$ corresponds to an even simple root; $\otimes$ to an odd isotropic simple root; - to an odd non-isotropic simple root, and $\times$ stands for $\circ$ or $\otimes$, depending on whether the simple root is even or odd. Note that the sub-diagrams $\bigcirc \stackrel{2}{\longleftarrow} \otimes \longrightarrow \otimes$ and $\bigcirc \stackrel{2}{\longleftarrow} \otimes \longrightarrow \bigcirc$ correspond respectively to the sub-matrices $\left[\begin{array}{ccc}2 & -1 & 0 \\ -2 & 0 & 1 \\ 0 & 1 & 0\end{array}\right]$ and $\left[\begin{array}{ccc}2 & -1 & 0 \\ -2 & 0 & 1 \\ 0 & -1 & 2\end{array}\right]$ in Cartan matrices.

For convenience, we take a slight variation of the usual definition [2, 15, 22] of quantised universal enveloping superalgebras (see Remark 2.5 below for further comments). Let us fix $q \in \mathbb{C}$ such that $q \neq 0, \pm 1$, and let $q^{1 / 2}$ be a fixed square root of $q$. Denote

$$
q_{i}=\left\{\begin{array}{ll}
q^{\frac{\left(\alpha_{i}, \alpha_{i}\right)}{2}}, & \text { if }\left(\alpha_{i}, \alpha_{i}\right) \neq 0 \\
q, & \text { if }\left(\alpha_{i}, \alpha_{i}\right)=0,
\end{array} \quad \theta_{i}= \begin{cases}1, & \text { if }\left|\left(\alpha_{i}, \alpha_{i}\right)\right|=1,2 \\
2, & \text { if }\left|\left(\alpha_{i}, \alpha_{i}\right)\right|=0,4\end{cases}\right.
$$

Note that $q_{i}^{a_{i j}}=q_{j}^{a_{j, i}}=q^{\left(\alpha_{i}, \alpha_{j}\right)}$. In what follows, $[x, y]_{v}=x y-(-1)^{[x][y]} v y x$.

Definition 2.4. The quantised universal enveloping superalgebra $\mathrm{U}_{q}(\mathfrak{g}, \Pi)$ of $\mathfrak{g}$ with the fundamental system $\Pi$ is an associative superalgebra over $\mathbb{C}$ with identity, which is defined by the following presentation: The generators are $e_{i}, f_{i}, k_{i}^{ \pm 1}$, where $e_{s}, f_{s},(s \in \tau)$, are odd and the rest are even, and the relations are given by

$$
\begin{gathered}
k_{i} k_{i}^{-1}=k_{i}^{-1} k_{i}=1, \quad k_{i} k_{j}=k_{j} k_{i}, \\
k_{i} e_{j} k_{i}^{-1}=q_{i}^{a_{i j}} e_{j}, \quad k_{i} f_{j} k_{i}^{-1}=q_{i}^{-a_{i j}} f_{j}, \\
e_{i} f_{j}-(-1)^{\left[e_{i}\right]\left[f_{j}\right]} f_{j} e_{i}=\delta_{i j} \frac{k_{i}-k_{i}^{-1}}{q^{\theta_{i}}-q^{-\theta_{i}}} ; \\
\text { if } a_{s s}=0, \quad\left(e_{s}\right)^{2}=\left(f_{s}\right)^{2}=0, \\
\text { if } a_{i i} \neq 0, i \neq j, \quad\left(\operatorname{Ad}_{e_{i}}\right)^{1-a_{i j}}\left(e_{j}\right)=\left(\operatorname{Ad}_{f_{i}}\right)^{1-a_{i j}}\left(f_{j}\right)=0,
\end{gathered}
$$

where $\operatorname{Ad}_{e_{i}}(x)$ and $\operatorname{Ad}_{f_{i}}(x)$ are defined by (2.3);

(3) and higher order Serre relations [16] (also see [21]) associated with the following subdiagrams of Dynkin diagrams

(A) $\underset{s-1}{\times} \underset{s}{\bigotimes} \underset{s+1}{\times}$ with $a_{s-1, s}=-a_{s, s+1}$, the associated higher order Serre relations are $\operatorname{Ad}_{e_{s}} \operatorname{Ad}_{e_{s-1}} \operatorname{Ad}_{e_{s}}\left(e_{s+1}\right)=0, \quad \operatorname{Ad}_{f_{s}} \operatorname{Ad}_{f_{s-1}} \operatorname{Ad}_{f_{s}}\left(f_{s+1}\right)=0$

(B) $\underset{s-1}{\longleftarrow} \underset{s}{\longrightarrow} \underset{s+1}{\longrightarrow}$, the associated higher order Serre elements are

$$
\operatorname{Ad}_{e_{s}} \operatorname{Ad}_{e_{s-1}} \operatorname{Ad}_{e_{s}}\left(e_{s+1}\right)=0, \quad \operatorname{Ad}_{f_{s}} \operatorname{Ad}_{f_{s-1}} \operatorname{Ad}_{f_{s}}\left(f_{s+1}\right)=0
$$


(C) $\underset{s-1}{x} \underset{s}{\longrightarrow} \longrightarrow \mathrm{O}_{s+1}$, the associated higher order Serre relations are $\operatorname{Ad}_{e_{s}} \operatorname{Ad}_{e_{s-1}} \operatorname{Ad}_{e_{s}}\left(e_{s+1}\right)=0, \quad \operatorname{Ad}_{f_{s}} \operatorname{Ad}_{f_{s-1}} \operatorname{Ad}_{f_{s}}\left(f_{s+1}\right)=0 ;$

(D) $\bigcirc \underset{s-1}{\stackrel{2}{2}} \bigotimes_{s} \longrightarrow \underset{s+1}{\longrightarrow}$, the associated higher order Serre relations are

$$
\begin{aligned}
& {\left[\operatorname{Ad}_{e_{s+1}}\left(e_{s}\right),\left[\operatorname{Ad}_{e_{s+1}}\left(e_{s}\right), \operatorname{Ad}_{e_{s}}\left(e_{s-1}\right)\right]_{v_{1}}\right]_{v_{2}}=0,} \\
& {\left[\operatorname{Ad}_{f_{s+1}}\left(e_{s}\right),\left[\operatorname{Ad}_{f_{s+1}}\left(f_{s}\right), \operatorname{Ad}_{f_{s}}\left(f_{s-1}\right)\right]_{v_{1}}\right]_{v_{2}}=0,}
\end{aligned}
$$

where $v_{1}=q^{-\left(\alpha_{s}, \alpha_{s+1}\right)}, v_{2}=q^{\left(\alpha_{s}, \alpha_{s+1}\right)}$;

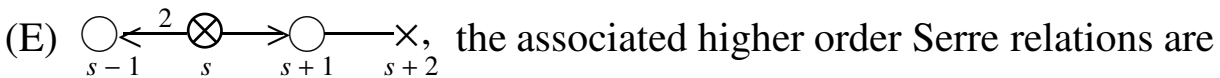

$$
\begin{aligned}
& {\left[\operatorname{Ad}_{e_{s+2}}\left(\operatorname{Ad}_{e_{s+1}} e_{s}\right),\left[\operatorname{Ad}_{e_{s+1}} e_{s}, \operatorname{Ad}_{e_{s}} e_{s-1}\right]_{v_{1}}\right]=0,} \\
& {\left[\operatorname{Ad}_{f_{s+2}}\left(\operatorname{Ad}_{f_{s+1}} f_{s}\right),\left[\operatorname{Ad}_{f_{s+1}} f_{s}, \operatorname{Ad}_{f_{s}} f_{s-1}\right]_{v_{1}}\right]=0, v_{1}=q^{-\left(\alpha_{s}, \alpha_{s+1}\right)} ;}
\end{aligned}
$$

(F)

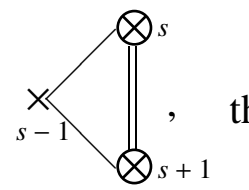

the associated higher order Serre relations are

$$
\begin{aligned}
& \operatorname{Ad}_{e_{s}} \operatorname{Ad}_{e_{s+1}}\left(e_{s-1}\right)-\operatorname{Ad}_{e_{s+1}} \operatorname{Ad}_{e_{s}}\left(e_{s-1}\right)=0, \\
& \operatorname{Ad}_{f_{s}} \operatorname{Ad}_{f_{s+1}}\left(f_{s-1}\right)-\operatorname{Ad}_{f_{s+1}} \operatorname{Ad}_{f_{s}}\left(f_{s-1}\right)=0 ;
\end{aligned}
$$

where $\operatorname{Ad}_{e_{i}}(x)$ and $\operatorname{Ad}_{f_{i}}(x)$ are defined by

$$
\operatorname{Ad}_{e_{i}}(x)=e_{i} x-(-1)^{\left[e_{i}\right][x]} k_{i} x k_{i}^{-1} e_{i}, \quad \operatorname{Ad}_{f_{i}}(x)=f_{i} x-(-1)^{\left[f_{i}\right][x]} k_{i}^{-1} x k_{i} f_{i} .
$$

Remark 2.5. We have used $\frac{k_{i}-k_{i}^{-1}}{q^{\theta_{i}}-q^{-\theta_{i}}}$ instead of the standard expression $\frac{k_{i}-k_{i}^{-1}}{q_{i}-q_{i}^{-1}}$ in the third relation of (1). A consequence is that $q^{ \pm 1 / 2}$ never appears in our definition of the quantised universal enveloping superalgebras.

Corresponding to each simple root $\alpha_{i} \in \Pi$ of $\mathfrak{g}$, we introduce a group $\mathbb{Z}_{2}$ generated by $\sigma_{i}$ such that $\sigma_{i}^{2}=1$, and let $G$ be the direct product of all such groups. Then $G=\mathbb{Z}_{2}^{\times|\Pi|}$, where $|\Pi|$ denotes the cardinality of $\Pi$. We define a G-action on $\mathrm{U}_{q}(\mathfrak{g}, \Pi)$ by

$$
\begin{aligned}
& \sigma_{i} \cdot e_{j}=(-1)^{\left(\alpha_{i}, \alpha_{j}\right)} e_{j}, \quad \sigma_{i} \cdot f_{j}=(-1)^{-\left(\alpha_{i}, \alpha_{j}\right)} f_{j}, \quad \sigma_{i} \cdot k_{j}=k_{j}, \quad i \neq 0, \\
& \sigma_{0} \cdot e_{j}=(-1)^{\delta_{i, 0}} e_{j}, \quad \sigma_{0} \cdot f_{j}=(-1)^{-\delta_{i, 0}} f_{j}, \quad \sigma_{0} \cdot k_{j}=k_{j}, \quad \forall j,
\end{aligned}
$$

where the second line is present only when $\mathfrak{g}$ is an affine superalgebra.

Definition 2.6. Let $\mathfrak{H}_{q}(\mathfrak{g}, \Pi)=\mathrm{U}_{q}(\mathfrak{g}, \Pi) \sharp \mathbb{C} G$ denote the smash product superalgebra with $\mathfrak{U}_{q}(\mathfrak{g}, \Pi)_{\overline{0}}=\mathrm{U}_{q}(\mathfrak{g}, \Pi)_{\overline{0}} \otimes \mathbb{C} G$ and $\mathfrak{U}_{q}(\mathfrak{g}, \Pi)_{\overline{1}}=\mathrm{U}_{q}(\mathfrak{g}, \Pi)_{\overline{1}} \otimes \mathbb{C} G$, where the multiplication is defined by

$$
(x \otimes \sigma)\left(x^{\prime} \otimes \sigma^{\prime}\right)=x\left(\sigma \cdot x^{\prime}\right) \otimes \sigma \sigma^{\prime}, \quad \forall x, x^{\prime} \in \mathrm{U}_{q}(\mathfrak{g}, \Pi), \sigma, \sigma^{\prime} \in \mathbb{C} \mathrm{G} .
$$


To simplify the notation, we write $x$ for $x \otimes 1$ for any $x \in \mathrm{U}_{q}(\mathfrak{g}, \Pi)$, and $\sigma$ for $1 \otimes \sigma$ for any $\sigma \in \mathbb{C} G$. Then in $\mathfrak{U}_{q}(\mathfrak{g}, \Pi)$, we have

$$
\begin{aligned}
& \sigma_{i} e_{j} \sigma_{i}^{-1}=(-1)^{\left(\alpha_{i}, \alpha_{j}\right)} e_{j}, \quad \sigma_{i} f_{j} \sigma_{i}^{-1}=(-1)^{-\left(\alpha_{i}, \alpha_{j}\right)} f_{j}, \quad \sigma_{i} k_{j} \sigma_{i}^{-1}=k_{j}, \quad i \neq 0, \\
& \sigma_{0} e_{j} \sigma_{0}^{-1}=(-1)^{\delta_{i, 0}} e_{j}, \quad \sigma_{0} f_{j} \sigma_{0}^{-1}=(-1)^{-\delta_{i, 0}} f_{j}, \quad \sigma_{0} k_{j} \sigma_{0}^{-1}=k_{j}, \quad \forall j .
\end{aligned}
$$

The quantised universal enveloping algebra $\mathrm{U}_{q}(\mathfrak{g}, \Pi)$ is a Hopf superalgebra, and the group algebra of $G$ has a canonical Hopf algebra structure. Their smash product inherits a Hopf superalgebra structure.

Proposition 2.7. The quantum superalgebra $\mathfrak{U}_{q}(\mathfrak{g}, \Pi)$ is a Hopf superalgebra with comultiplication $\Delta: \mathfrak{U}_{q}(\mathfrak{g}, \Pi) \rightarrow \mathfrak{U}_{q}(\mathfrak{g}, \Pi) \otimes \mathfrak{U}_{q}(\mathfrak{g}, \Pi)$,

$$
\begin{array}{cl}
\Delta\left(e_{i}\right)=e_{i} \otimes 1+k_{i} \otimes e_{i}, & \Delta\left(f_{i}\right)=f_{i} \otimes k_{i}^{-1}+1 \otimes f_{i}, \\
\Delta\left(k_{i}\right)=k_{i} \otimes k_{i}, & \Delta\left(\sigma_{i}\right)=\sigma_{i} \otimes \sigma_{i} ;
\end{array}
$$

counit $\varepsilon: \mathfrak{U}_{q}(\mathfrak{g}, \Pi) \rightarrow \mathbb{C}, \quad \varepsilon\left(e_{i}\right)=\varepsilon\left(f_{i}\right)=0, \quad \varepsilon\left(k_{i}^{ \pm 1}\right)=1, \quad \varepsilon\left(\sigma_{i}\right)=1 ;$ and antipode $S: \mathfrak{U}_{q}(\mathfrak{g}, \Pi) \rightarrow \mathfrak{U}_{q}(\mathfrak{g}, \Pi), \quad S\left(e_{i}\right)=-k_{i}^{-1} e_{i}, \quad S\left(f_{i}\right)=-f_{i} k_{i}, \quad S\left(k_{i}\right)=k_{i}^{-1}, \quad S\left(\sigma_{i}\right)=\sigma_{i}^{-1}$.

\section{Algebraic ISOMORPhisms}

We prove Theorem 1.1 in this section. The proof requires detailed considerations of the structures of the relevant quantum superalgebras, thus is very lengthy as each pair $\left(\mathfrak{g}, \mathfrak{g}^{\prime}\right)$ involves numerous cases corresponding to different choices of fundamental systems. We will present only the main steps of the proof, omitting most of the detailed calculations.

Let $\left(\mathfrak{g}, \mathfrak{g}^{\prime}\right)$ be a pair of Lie superalgebras or affine Lie superalgebras in Theorem 1.1 . Choose any fundamental system $\Pi$ for $\mathfrak{g}$ with $\tau$ being the labelling set for the odd simple roots. By Lemma 2.2, $\Pi^{\prime}=\phi(\Pi)$ is the corresponding fundamental system of $\mathfrak{g}^{\prime}$ with the labelling set $\tau^{\prime}$ for the odd simple roots. We write $\alpha_{i}^{\prime}=\phi\left(\alpha_{i}\right)$ for the simple roots of $\mathfrak{g}^{\prime}$. Note that $\alpha_{s}^{\prime} \in \Pi^{\prime}$ is isotropic if and only if $\alpha_{s} \in \Pi$ is.

Let $\left\{e_{i}, f_{i}, k_{i}^{ \pm 1}, \sigma_{i}\right\}$ be the set of generators of the quantum superalgebra $\mathfrak{U}_{q}(\mathfrak{g}, \Pi)$, and denote by $\mathrm{G}$ the group generated by the elements $\sigma_{i}$. Similarly, we let $\left\{e_{i}^{\prime}, f_{i}^{\prime}, k_{i}^{\prime \pm 1}, \sigma_{i}^{\prime}\right\}$ be the standard generating set of $\mathfrak{U}_{-q}\left(\mathfrak{g}^{\prime}, \Pi^{\prime}\right)$, and denote by $\mathrm{G}^{\prime}$ the group generated by the elements $\sigma_{i}^{\prime}$.

For $1 \leq i \leq m+n$, we introduce the following elements

$$
\begin{aligned}
& \Phi_{i}=\prod_{k=i}^{m+n} \sigma_{k}, \quad \tilde{\Phi}_{i}=\prod_{k=0}^{m+n} \sigma_{i+2 k}, \quad \text { in } \mathfrak{U}_{q}(\mathfrak{g}, \Pi) \\
& \Phi_{i}^{\prime}=\prod_{k=i}^{m+n} \sigma_{k}^{\prime}, \quad \tilde{\Phi}_{i}^{\prime}=\prod_{k=0}^{m+n} \sigma_{i+2 k}^{\prime}, \quad \text { in } \mathfrak{U}_{-q}\left(\mathfrak{g}^{\prime}, \Pi^{\prime}\right) \text {, }
\end{aligned}
$$

where $\sigma_{j} \in G$ and $\sigma_{j}^{\prime} \in G^{\prime}$ are both 1 if $j \geq m+n+1$. Note that

$$
\Phi_{m+n}=\tilde{\Phi}_{m+n}=\sigma_{m+n}, \quad \Phi_{m+n}^{\prime}=\tilde{\Phi}_{m+n}^{\prime}=\sigma_{m+n}^{\prime} .
$$


For $i=1,2, \ldots, m+n$, we define the following elements

$$
\begin{aligned}
& E_{i}=\Phi_{i+1} e_{i}, \quad F_{i}=\Phi_{i} f_{i}, \quad i \notin \tau, \\
& E_{i}=\tilde{\Phi}_{i+2} e_{i}, \quad F_{i}=\tilde{\Phi}_{i} f_{i}, \quad i \in \tau, \\
& K_{i}=\sigma_{i} k_{i}, \quad \text { which belong to } \mathfrak{U}_{q}(\mathfrak{g}, \Pi) ; \text { and } \\
& E_{i}^{\prime}=\Phi_{i+1}^{\prime} e_{i}^{\prime}, \quad F_{i}^{\prime}=\Phi_{i}^{\prime} f_{i}^{\prime}, \quad i \notin \tau^{\prime}, \\
& E_{i}^{\prime}=\tilde{\Phi}_{i+2}^{\prime} e_{i}^{\prime}, \quad F_{i}^{\prime}=\tilde{\Phi}_{i}^{\prime} f_{i}^{\prime}, \quad i \in \tau^{\prime}, \\
& K_{i}^{\prime}=\sigma_{i}^{\prime} k_{i}^{\prime}, \quad \text { which belong to } \mathfrak{U}_{-q}\left(\mathfrak{g}^{\prime}, \Pi^{\prime}\right),
\end{aligned}
$$

where $\Phi_{m+n+k}=\tilde{\Phi}_{m+n+k}=1$ and $\Phi_{m+n+k}^{\prime}=\tilde{\Phi}_{m+n+k}^{\prime}=1$ for all $k>0$.

If $\left(\mathfrak{g}, \mathfrak{g}^{\prime}\right)$ is a pair of affine Lie superalgebras, we will also define elements

$$
E_{0}, F_{0}, K_{0} \in \mathfrak{U}_{q}(\mathfrak{g}, \Pi), \quad \text { and } \quad E_{0}^{\prime}, F_{0}^{\prime}, K_{0}^{\prime} \in \mathfrak{U}_{-q}\left(\mathfrak{g}^{\prime}, \Pi^{\prime}\right),
$$

the explicit expressions of which depend on the affine Lie superalgebras and fundamental systems, and will be given in the proof of the following result.

Theorem 3.1. The associative algebra isomorphism $\mathfrak{U}_{-q}\left(\mathfrak{g}^{\prime}, \Pi^{\prime}\right) \stackrel{\cong}{\longrightarrow} \mathfrak{U}_{q}(\mathfrak{g}, \Pi)$ of Theorem 1.1 is given by

$$
\sigma_{i}^{\prime} \mapsto \sigma_{i}, \quad e_{i}^{\prime} \mapsto E_{i}, \quad f_{i}^{\prime} \mapsto F_{i}, \quad k_{i}^{\prime} \mapsto K_{i}, \quad \forall i
$$

with the inverse map

$$
\sigma_{i} \mapsto \sigma_{i}^{\prime}, \quad e_{i} \mapsto E_{i}^{\prime}, \quad f_{i} \mapsto F_{i}^{\prime}, \quad k_{i} \mapsto K_{i}^{\prime}, \quad \forall i
$$

This is a more explicit version of Theorem 1.1. If we can prove that the maps (3.2) and (3.3) are algebra homomorphisms, then we immediately see that they are inverses of each other since $\Phi_{i}^{2}=1$ for all $i$. It is clear from equation (2.4) that the maps preserve the action of $G$ on $\mathrm{U}_{\mathrm{q}}(\mathfrak{g}, \Pi)$ and the action of $G^{\prime}$ on $\mathrm{U}_{-q}\left(\mathfrak{g}^{\prime}, \Pi^{\prime}\right)$. Thus what remains to be shown is that

- $E_{i}, F_{i}, K_{i}^{ \pm 1}$ satisfy the defining relations of $\mathrm{U}_{-q}\left(\mathfrak{g}^{\prime}, \Pi^{\prime}\right)$ obeyed by the standard generators $e_{i}^{\prime}, f_{i}^{\prime}, k_{i}^{\prime 11}(1 \leq i \leq m+n)$; and

- $E_{i}^{\prime}, F_{i}^{\prime}, K_{i}^{\prime \pm 1}$ satisfy the defining relations of $\mathrm{U}_{q}(\mathfrak{g}, \Pi)$ obeyed by the standard generators $e_{i}, f_{i}, k_{i}^{ \pm 1}(1 \leq i \leq m+n)$.

The proof simply boils down to deducing the desired relations satisfied by $E_{i}, F_{i}, K_{i}^{ \pm 1}$ (rsep. $E_{i}^{\prime}, F_{i}^{\prime}, K_{i}^{\prime \pm 1}$ ), from the defining relations of $\mathrm{U}_{q}(\mathfrak{g}, \Pi)$ (resp. $\mathrm{U}_{-q}\left(\mathfrak{g}^{\prime}, \Pi^{\prime}\right)$ ). The proofs for the two statements are exactly the same, thus we will only present the details for the first one, which will occupy the next three sections. The following notation will be used,

$$
\begin{aligned}
& {[k]_{z}=\frac{z^{k}-z^{-k}}{z-z^{-1}}, \quad\{k\}_{z}=\frac{z^{k}-(-z)^{-k}}{z+z^{-1}}, \quad \text { for } k \in \mathbb{Z},} \\
& {[0]_{z} !=\{0\}_{z} !=1, \quad[N]_{z} !=\prod_{i=1}^{N}[i]_{z}, \quad\{N\}_{z} !=\prod_{i=1}^{N}\{i\}_{z}, \text { for } \quad 1 \leq N \in \mathbb{N},} \\
& {\left[\begin{array}{c}
N \\
k
\end{array}\right]_{z}=\frac{[N]_{z} !}{[N-k]_{z} ![k]_{z} !}, \quad\left\{\begin{array}{c}
N \\
k
\end{array}\right\}_{z}=\frac{\{N\}_{z} !}{\{N-k\}_{z} !\{k\}_{z} !}, \quad \text { for } k \leq N \in \mathbb{N},}
\end{aligned}
$$

where $z \in \mathbb{C}$ such that the expressions above are defined. 
3.1. The case of $\mathfrak{d s p}(2 m+1 \mid 2 n)$ and $\mathfrak{d s p}(2 n+1 \mid 2 m)$. Recall from Section 2.1 that the ambient space of the roots of $\mathfrak{g}=\mathfrak{D} \mathfrak{s p}(2 m+1 \mid 2 n)$ is $\mathcal{E}(m \mid n)$. Each admissible ordered basis of it leads to a fundamental system $\Pi=\left\{\alpha_{i} \mid 1 \leq i \leq m+n\right\}$ with the $\alpha_{i}$ given in Table 2, Now $\mathfrak{g}^{\prime}=\mathfrak{D s p}(2 n+1 \mid 2 m)$ with the corresponding fundamental system $\Pi^{\prime}=\left\{\alpha_{i}^{\prime}=\phi\left(\alpha_{i}\right) \mid\right.$ $i=1,2, \ldots, m+n\}$. The ambient space of the roots is $\mathcal{E}(n \mid m)$. In the case $\alpha_{m+n}=\delta_{n} \in \Pi$, which is odd, $\alpha_{m+n}^{\prime}=\varepsilon_{n}$ is an even simple root in $\Pi^{\prime}$, and the Dynkin diagrams of $\Pi$ and $\Pi^{\prime}$ are the Type (1) diagrams in Table 4. In this case, $\tau^{\prime}=\tau \backslash\{m+n\}$. If $\alpha_{m+n}=\varepsilon_{m} \in \Pi$, which is even, $\alpha_{m+n}^{\prime}=\delta_{m}$ is an odd simple root in $\Pi^{\prime}$, and the Dynkin diagrams of $\Pi$ and $\Pi^{\prime}$ are the Type (2) diagrams in Table 4. In this case, $\tau=\tau^{\prime} \backslash\{m+n\}$.

TABLE 4. Dynkin diagram of $\mathfrak{p s p}(2 m+1 \mid 2 n)$ and $\mathfrak{o s p}(2 n+1 \mid 2 m)$

\begin{tabular}{|c|c|c|c|c|}
\hline Type & \multicolumn{2}{|c|}{$\mathfrak{g}=\mathfrak{v} \mathfrak{s p}(2 m+1 \mid 2 n)$} & \multicolumn{2}{|c|}{$\mathfrak{g}^{\prime}=\mathfrak{D} \mathfrak{s p}(2 n+1 \mid 2 m)$} \\
\hline (1) & $\begin{array}{ll}x & \times- \\
\alpha_{1} & \alpha_{2} \\
\end{array}$ & $\underset{\alpha_{m+n-1}}{\longrightarrow}$ & $\underset{\alpha_{1}^{\prime}}{\times}{ }_{\alpha_{2}^{\prime}}^{\times-}$ & $\underset{\alpha_{m+n-1}^{\prime}}{ } \times$ \\
\hline (2) & $\begin{array}{ll}\times & \times- \\
\alpha_{1} & \alpha_{2}\end{array}$ & $\underset{\alpha_{m+n-1}}{\Longrightarrow}$ & $\underset{\alpha_{1}^{\prime}}{\longleftarrow} \underset{\alpha_{2}^{\prime}}{X-}$ & $\alpha_{m+n-1}^{\prime}$ \\
\hline
\end{tabular}

The quantum superalgebra $\mathfrak{U}_{q}(\mathfrak{D s p}(2 m+1 \mid 2 n), \Pi)$ is generated by $\mathrm{U}_{\mathrm{q}}(\mathfrak{D s p}(2 m+1 \mid 2 n), \Pi)$, and the elements $\sigma_{i}$, which generate a group $G=\mathbb{Z}_{2}^{\times(m+n)}$. The commutation relations of the $\sigma_{i}$ with the generators of $\mathrm{U}_{\mathrm{q}}(\mathfrak{D s p}(2 m+1 \mid 2 n), \Pi)$ are given by (2.4). The quantum superalgebra $\mathfrak{U}_{-q}\left(\mathfrak{D s p}(2 n \mid 2 m+1), \Pi^{\prime}\right)$ can be described similarly.

Let $t=-q$, and let $t^{1 / 2}$ be a square root for $t$. Denote $t_{i}=t^{\left(\alpha_{i}, \alpha_{i}\right) / 2}$. Note that relations among $E_{i}, F_{i}, K_{i}$ depend on $t^{ \pm 1}$, but not on $t^{ \pm 1 / 2}$.

Proof of Theorem 3.1 for $\left(\mathfrak{g}, \mathfrak{g}^{\prime}\right)=(\mathfrak{D} \mathfrak{p p}(2 m+1 \mid 2 n), \mathfrak{b} \mathfrak{p}(2 n+1 \mid 2 m))$. Consider first the Type (1) Dynkin diagrams in Table 4. In this case, $\alpha_{m+n}=\delta_{n} \in \Pi$. Let $\bar{i}=1$ if $i \in \tau^{\prime}$ and 0 otherwise. Then it is easy to see that

$$
E_{i} F_{j}-(-1)^{\bar{i} \bar{j}} F_{j} E_{i}=\delta_{i j} \frac{K_{i}-K_{i}^{-1}}{t^{\theta_{i}}-t^{-\theta_{i}}}, \quad 1 \leq i, j \leq m+n .
$$

For example,

$$
\begin{aligned}
& E_{m+n} F_{m+n}-F_{m+n} E_{m+n}=-\sigma_{m+n}\left(e_{m+n} f_{m+n}+f_{m+n} e_{m+n}\right)=\frac{K_{m+n}-K_{m+n}^{-1}}{t-t^{-1}}, \\
& E_{k} F_{m+n}-F_{m+n} E_{k}=(-1)^{\delta_{k, m+n-1}} \tilde{\Phi}_{k+2} \tilde{\Phi}_{m+n}\left(e_{k} f_{m+n}+f_{m+n} e_{k}\right)=0, \quad k \in \tau^{\prime} .
\end{aligned}
$$

The other relations listed in (1) of Definition 2.4 can be proved in exactly the same way.

We now consider the Serre relations (2) in Definition 2.4, which lead to

$$
E_{i}^{2}=F_{i}^{2}=0, \quad \text { if } \alpha_{i} \in \Pi \text { is isotropic, }
$$

$$
\begin{aligned}
& \sum_{k=0}^{1-a_{i j}}(-1)^{k}\left[\begin{array}{c}
1-a_{i j} \\
k
\end{array}\right]_{t_{i}} E_{i}^{k} E_{j} E_{i}^{1-a_{i j}-k}=0, \\
& \sum_{k=0}^{1-a_{i j}}(-1)^{k}\left[\begin{array}{c}
1-a_{i j} \\
k
\end{array}\right]_{t_{i}} F_{i}^{k} F_{j} F_{i}^{1-a_{i j}-k}=0, \quad i \notin \tau^{\prime}, i \neq j .
\end{aligned}
$$


To see this, we consider, for example, the case $i=m+n$ and $j=m+n-1 \notin \tau$. We have

$$
\sum_{k=0}^{3}(-1)^{k}\left[\begin{array}{l}
3 \\
k
\end{array}\right]_{t_{m+n}} E_{m+n}^{k} E_{m+n-1} E_{m+n}^{3-k}=\Phi_{m+n} \sum_{k=0}^{3}(-1)^{\frac{k(k+1)}{2}}\left\{\begin{array}{l}
3 \\
k
\end{array}\right\}_{q_{m+n}} e_{m+n}^{k} e_{m+n-1} e_{m+n}^{3-k}=0,
$$

where we have used $\left[\begin{array}{l}3 \\ k\end{array}\right]_{t_{m+n}}=(-1)^{\frac{k(k+1)}{2}}\left\{\begin{array}{l}3 \\ k\end{array}\right\}_{q_{m+n}}$ for $0 \leq k \leq 3$. For $m+n-1 \in \tau$, the above equation remains valid if we replace the expression between the equality signs by $\sum_{k=0}^{3}(-1)^{\frac{k(k-1)}{2}}\left\{\begin{array}{l}3 \\ k\end{array}\right\}_{q_{m+n}} e_{m+n}^{k} e_{m+n-1} e_{m+n}^{3-k}$.

Higher order Serre relations can arise from two types of sub-diagrams only in the present case. Denote

$$
\begin{aligned}
e_{i ; s ; j}= & e_{i}\left(e_{s} e_{j}-(-1)^{\left[e_{j}\right]} q_{j}^{a_{j s}} e_{j} e_{s}\right) \\
& -(-1)^{\left[e_{i}\right]\left(1+\left[e_{j}\right]\right)} q_{i}^{a_{i s}+a_{i j}}\left(e_{s} e_{j}-(-1)^{\left[e_{j}\right]} q_{j}^{a_{j s}} e_{j} e_{s}\right) e_{i}, \\
f_{i ; s ; j}= & f_{i}\left(f_{s} f_{j}-(-1)^{\left[f_{j}\right]} q_{j}^{a_{j s}} f_{j} f_{s}\right) \\
& -(-1)^{\left[f_{i}\right]\left(1+\left[f_{j}\right]\right)} q_{i}^{a_{i s}+a_{i j}}\left(f_{s} f_{j}-(-1)^{\left[f_{j}\right]} q_{j}^{a_{j s}} f_{j} f_{s}\right) f_{i} .
\end{aligned}
$$

Case 1. $\underset{s-1}{\longrightarrow} \bigotimes_{s} \underset{s+1}{x}$, with the associated higher order Serre relations given by

$$
\begin{aligned}
& e_{s} e_{s-1 ; s ; s+1}+(-1)^{\left[e_{s-1}\right]+\left[e_{s+1}\right]} e_{s-1 ; s ; s+1} e_{s}=0, \\
& f_{s} f_{s-1 ; s ; s+1}+(-1)^{\left[f_{s-1}\right]+\left[f_{s+1}\right]} f_{s-1 ; s ; s+1} f_{s}=0 .
\end{aligned}
$$

Let us first assume that $s-1, s+1 \notin \tau$. In this case, $s \neq m+n-1, a_{s-1, s}=a_{s+1, s}=-1$ and $q_{s+1}=q_{s-1}^{-1}$, with $q_{s-1}=q$ or $q^{-1}$ depending on depending on the value of $\theta$ in (2.1). Then (3.7) is given by

$$
\begin{aligned}
& e_{s} e_{s-1 ; s ; s+1}+e_{s-1 ; s ; s+1} e_{s}=0, \quad f_{s} f_{s-1 ; s ; s+1}+f_{s-1 ; s ; s+1} f_{s}=0, \quad \text { with } \\
& e_{s-1 ; s ; s+1}=e_{s-1}\left(e_{s} e_{s+1}-q_{s+1}^{-1} e_{s+1} e_{s}\right)-q_{s-1}^{-1}\left(e_{s} e_{s+1}-q_{s+1}^{-1} e_{s+1} e_{s}\right) e_{s-1}, \\
& f_{s-1 ; s ; s+1}=f_{s-1}\left(f_{s} f_{s+1}-q_{s+1}^{-1} f_{s+1} f_{s}\right)-q_{s-1}^{-1}\left(f_{s} f_{s+1}-q_{s+1}^{-1} f_{s+1} f_{s}\right) f_{s-1} .
\end{aligned}
$$

Write $t_{s \pm 1}=-q_{s \pm 1}$, and let

$$
\begin{aligned}
& E_{s-1 ; s ; s+1}:=E_{s-1}\left(E_{s} E_{s+1}-t_{s+1}^{-1} E_{s+1} E_{s}\right)-t_{s-1}^{-1}\left(E_{s} E_{s+1}-t_{s+1}^{-1} E_{s+1} E_{s}\right) E_{s-1}, \\
& F_{s-1 ; s ; s+1}:=F_{s-1}\left(F_{s} F_{s+1}-t_{s+1}^{-1} F_{s+1} F_{s}\right)-t_{s-1}^{-1}\left(F_{s} F_{s+1}-t_{s+1}^{-1} F_{s+1} F_{s}\right) F_{s-1} .
\end{aligned}
$$

For any mutually distinct $i, j, k$ not in $\tau$,

$$
E_{i} E_{j} E_{k}=(-1)^{\delta_{i, k+1}+\delta_{j, k+1}+\delta_{i, j+1}} \Phi_{i+1} \Phi_{j+1} \Phi_{k+1} e_{i} e_{j} e_{k} .
$$

If any one of $i, j, k$ is in $\tau$, say, $j \in \tau$, the identity still holds if we replace $\Phi_{j+1}$ by $\tilde{\Phi}_{j+2}$. There are also similar relations for $F$ 's. Using these facts, we obtain

$$
E_{s-1 ; s ; s+1}=\Phi_{s} \tilde{\Phi}_{s+2} \Phi_{s+2} e_{s-1 ; s ; s+1}, \quad F_{s-1 ; s ; s+1}=\Phi_{s-1} \tilde{\Phi}_{s} \Phi_{s+1} f_{s-1 ; s ; s+1} .
$$

This immediately leads to

$$
\begin{aligned}
& E_{s} E_{s-1 ; s ; s+1}+E_{s-1 ; s ; s+1} E_{s}=-\sigma_{s} \sigma_{s+1}\left(e_{s} e_{s-1 ; s ; s+1}+e_{s-1 ; s ; s+1} e_{s}\right)=0, \\
& F_{s} F_{s-1 ; s ; s+1}+F_{s-1 ; s ; s+1} F_{s}=-\sigma_{s-1} \sigma_{s}\left(f_{s} f_{s-1 ; s ; s+1}+f_{s-1 ; s ; s+1} f_{s}\right)=0 .
\end{aligned}
$$

Without assuming $s-1, s+1 \notin \tau$, we can still show that similar relations hold. 
In summary, for $s$ such that $\alpha_{s}$ is isotropic, we have

$$
\begin{aligned}
& E_{s} E_{s-1 ; s ; s+1}-(-1)^{1+\overline{s-1}+\overline{s+1}} E_{s-1 ; s ; s+1} E_{s}=0, \\
& F_{s} F_{s-1 ; s ; s+1}-(-1)^{1+\overline{s-1}+\overline{s+1}} F_{s-1 ; s ; s+1} F_{s}=0,
\end{aligned}
$$

where $\overline{s-1}$ and $\overline{s+1}$ are as in equation (3.4).

Case 2. $\underset{s-1}{\times} \underset{s}{\bigotimes} \Longrightarrow \bigcirc_{s+1}$, with the associated higher order Serre relations given by

$$
\begin{aligned}
& e_{s} e_{s-1 ; s ; s+1}-(-1)^{\left[e_{s-1}\right]} e_{s-1 ; s ; s+1} e_{s}=0, \\
& f_{s} f_{s-1 ; s ; s+1}-(-1)^{\left[f_{s-1}\right]} f_{s-1 ; s ; s+1} f_{s}=0 ;
\end{aligned}
$$

where $s+1=m+n$. By the similar method above, we have

$$
\begin{aligned}
& E_{s} E_{s-1 ; s ; s+1}-(-1)^{1+\overline{s-1}} E_{s-1 ; s ; s+1} E_{s}=0, \\
& F_{s} F_{s-1 ; s ; s+1}-(-1)^{1+\overline{s-1}} F_{s-1 ; s ; s+1} F_{s}=0 .
\end{aligned}
$$

Note that equations, (3.4), (3.5), (3.8) and (3.10) are the same as the defining relations of $\mathrm{U}_{-q}\left(\mathfrak{g}^{\prime}, \Pi^{\prime}\right)$ satisfied by the generators $e_{i}^{\prime}, f_{i}^{\prime}, k_{i}^{\prime}$. Thus we have shown that the map $\mathfrak{U}_{-q}\left(\mathfrak{g}^{\prime}, \Pi^{\prime}\right) \longrightarrow \mathfrak{U}_{q}(\mathfrak{g}, \Pi)$ given by $(\underline{3.2})$ is indeed an algebra homomorphism if $\alpha_{m+n}=\delta_{n}$, i.e., in the case of the Type (1) diagrams in Table 4. Similarly we can prove this for Type (2) diagrams in Table 4, where $\alpha_{m+n}=\varepsilon_{m}$.

3.2. The case of $\mathfrak{s l}(2 m+1 \mid 2 n)^{(2)}$ and $\mathfrak{p s p}(2 n+1 \mid 2 m)^{(1)}$. Given a fundamental system $\Pi=$ $\left\{\alpha_{i} \mid i=0,1, \ldots, m+n\right\}$ of $\mathfrak{g}=\mathfrak{s l}(2 m+1 \mid 2 n)^{(2)}$, we obtain a corresponding fundamental system $\Pi^{\prime}=\left\{\alpha_{i}^{\prime}=\phi\left(\alpha_{i}\right) \mid i=0,1, \ldots, m+n\right\}$ of $\mathfrak{g}^{\prime}=\mathfrak{o s p}(2 n+1 \mid 2 m)^{(1)}$ by Lemma 2.2. We draw the Dynkin diagrams for $\Pi$ and $\Pi^{\prime}$ in a row of Table 5, with the diagram for $\Pi$ on the left. The Dynkin diagrams corresponding to different choices of fundamental systems are divided into four types in the table.

Proof of Theorem 3.1 for $\left(\mathfrak{g}, \mathfrak{g}^{\prime}\right)=\left(\mathfrak{s l}(2 m+1 \mid 2 n)^{(2)}, \mathfrak{D s p}(2 n+1 \mid 2 m)^{(1)}\right)$. For $1 \leq i \leq m+$ $n$, we define $E_{i}, F_{i}, K_{i}, E_{i}^{\prime}, F_{i}^{\prime}, K_{i}^{\prime}$ by (3.1). Note that when the nodes of $\alpha_{0}$ and $\alpha_{0}^{\prime}$ are removed, the Dynkin diagrams in Table 5 reduce to the Dynkin diagrams for the finite dimensional Lie superalgebras $\mathfrak{v} \mathfrak{p p}(2 m+1 \mid 2 n)$ and $\mathfrak{v} \mathfrak{s p}(2 n+1 \mid 2 m)$. Thus by (3.1), the same reasoning in Section 3.1 can show that the elements $E_{i}, F_{i}, K_{i}$ (resp. $E_{i}^{\prime}, F_{i}^{\prime}, K_{i}^{\prime}$ ) for $1 \leq i \leq m+n$ have the desired properties.

What remains to be done, in order to complete the proof of Theorem 3.1, is to construct elements $E_{0}, F_{0}, K_{0} \in \mathfrak{U}_{q}(\mathfrak{g}, \Pi)$ (resp. $E_{0}^{\prime}, F_{0}^{\prime}, K_{0}^{\prime} \in \mathfrak{U}_{t}\left(\mathfrak{g}^{\prime}, \Pi^{\prime}\right)$ ), which satisfy the commutation relations obeyed by $e_{0}^{\prime}, f_{0}^{\prime}, k_{0}^{\prime}\left(\operatorname{resp} e_{0}, f_{0}, k_{0}\right)$. We do this for each of the four types of diagrams in Table 5. The proofs for $E_{0}, F_{0}, K_{0}$ and for $E_{0}^{\prime}, F_{0}^{\prime}, K_{0}^{\prime}$ are similar to those in Section 3.1, thus we will give the constructions for these elements only.

Case 1. Type (1) and Type (2) Dynkin diagrams in Table 5 .

$$
E_{0}=e_{0}, \quad F_{0}=f_{0}, \quad K_{0}=k_{0}, \text { and, } E_{0}^{\prime}=e_{0}^{\prime}, \quad F_{0}^{\prime}=f_{0}^{\prime}, \quad K_{0}^{\prime}=k_{0}^{\prime} .
$$

Note that for Type (2) Dynkin diagrams with $m+n>2$, the higher order Serre relations involving $e_{0}$ or $f_{0}$ are either of type (D) (for $2 \in \tau$ ) or (E) (for $2 \notin \tau$ ) (see Definition 2.4).

Case 2. Type (3) Dynkin diagrams in Table 5. In this case,

$$
\begin{array}{lll}
E_{0}=\Phi_{2} e_{0}, & F_{0}=\Phi_{1} f_{0}, & K_{0}=\sigma_{1} k_{0}, \\
E_{0}^{\prime}=\Phi_{2}^{\prime} e_{0}^{\prime}, & F_{0}^{\prime}=\Phi_{1}^{\prime} f_{0}^{\prime}, & K_{0}^{\prime}=\sigma_{1}^{\prime} k_{0}^{\prime} .
\end{array}
$$


TABLE 5. Dynkin diagrams of $\mathfrak{s l}(2 m+1 \mid 2 n)^{(2)}$ and $\mathfrak{D} \mathfrak{s p}(2 n+1 \mid 2 m)^{(1)}$

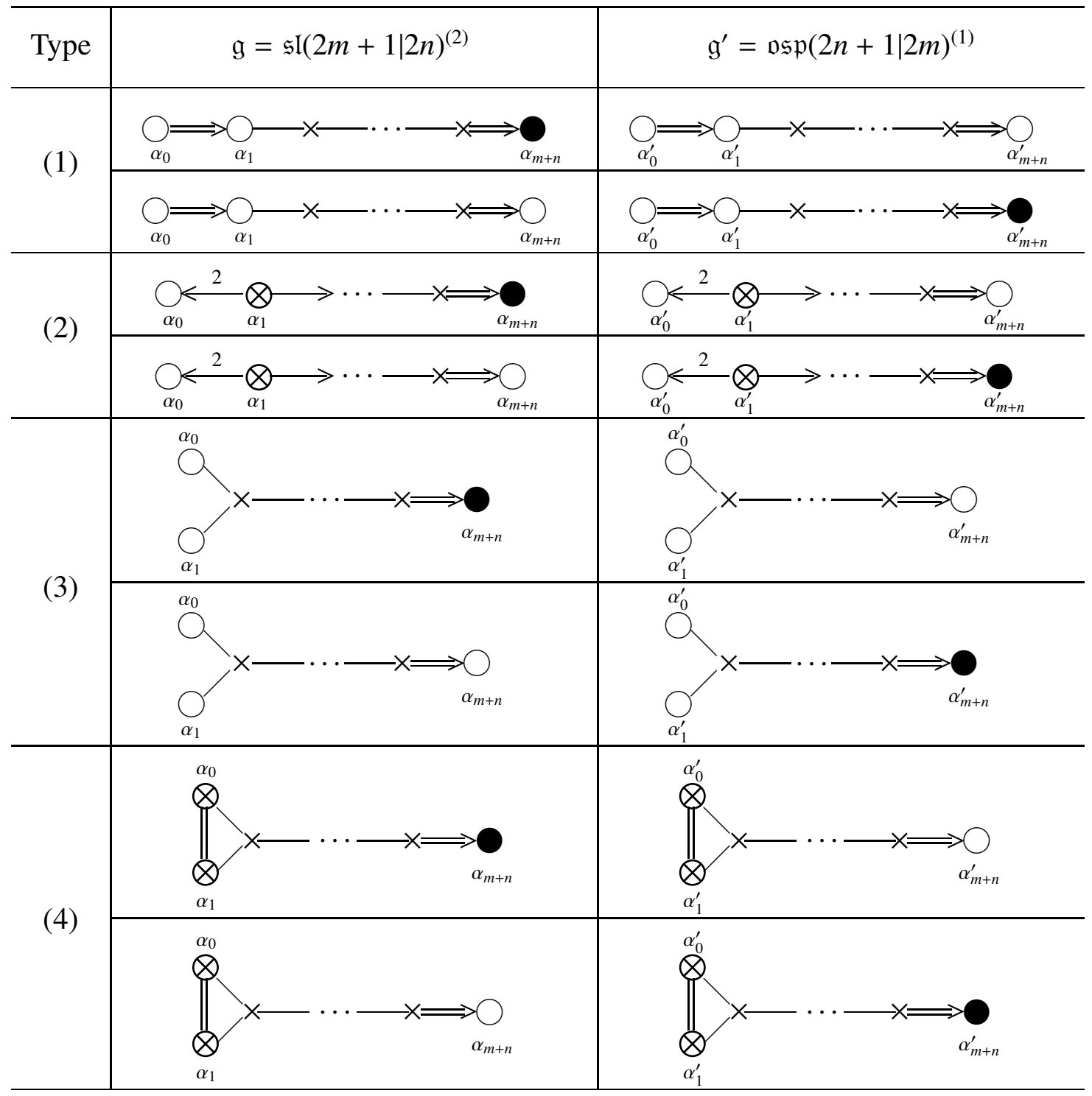

Case 3. Type (4) Dynkin diagrams in Table 5. This time $\alpha_{0}$ is an odd simple root. Define

$$
\begin{array}{lll}
E_{0}=\tilde{\Phi}_{3} e_{0}, & F_{0}=\tilde{\Phi}_{1} f_{0}, & K_{0}=\sigma_{1} k_{0}, \\
E_{0}^{\prime}=\tilde{\Phi}_{3}^{\prime} e_{0}^{\prime}, & F_{0}^{\prime}=\tilde{\Phi}_{1}^{\prime} f_{0}^{\prime}, & K_{0}^{\prime}=\sigma_{1}^{\prime} k_{0}^{\prime} .
\end{array}
$$

3.3. The case of $\mathfrak{o s p}(2 m+2 \mid 2 n)^{(2)}$ and $\mathfrak{o s p}(2 n+2 \mid 2 m)^{(2)}$. The Dynkin diagrams of $\mathfrak{g}=$ $\mathfrak{v} \mathfrak{s p}(2 m+2 \mid 2 n)^{(2)}$ and $\mathfrak{g}^{\prime}=\mathfrak{v} \mathfrak{s p}(2 n+2 \mid 2 m)^{(2)}$ are given in Table 6, where the diagrams for a fundamental system $\Pi$ of $\mathfrak{g}$ and the corresponding fundamental system $\Pi^{\prime}$ of $\mathfrak{g}^{\prime}$ are shown in the same row.

Proof of Theorem 3.1 for $\left(\mathfrak{g}, \mathfrak{g}^{\prime}\right)=\left(\mathfrak{D s p}(2 m+2 \mid 2 n)^{(2)}, \mathfrak{D s p}(2 n+2 \mid 2 m)^{(2)}\right)$. We will merely construct the elements $E_{i}, F_{i}, K_{i}, E_{i}^{\prime}, F_{i}^{\prime}, K_{i}^{\prime}$ here, as the proof of Theorem 3.1 is much 
TABLE 6. Dynkin diagrams of $\mathfrak{o s p}(2 m+2 \mid 2 n)^{(2)}$ and $\mathfrak{o s p}(2 n+2 \mid 2 m)^{(2)}$

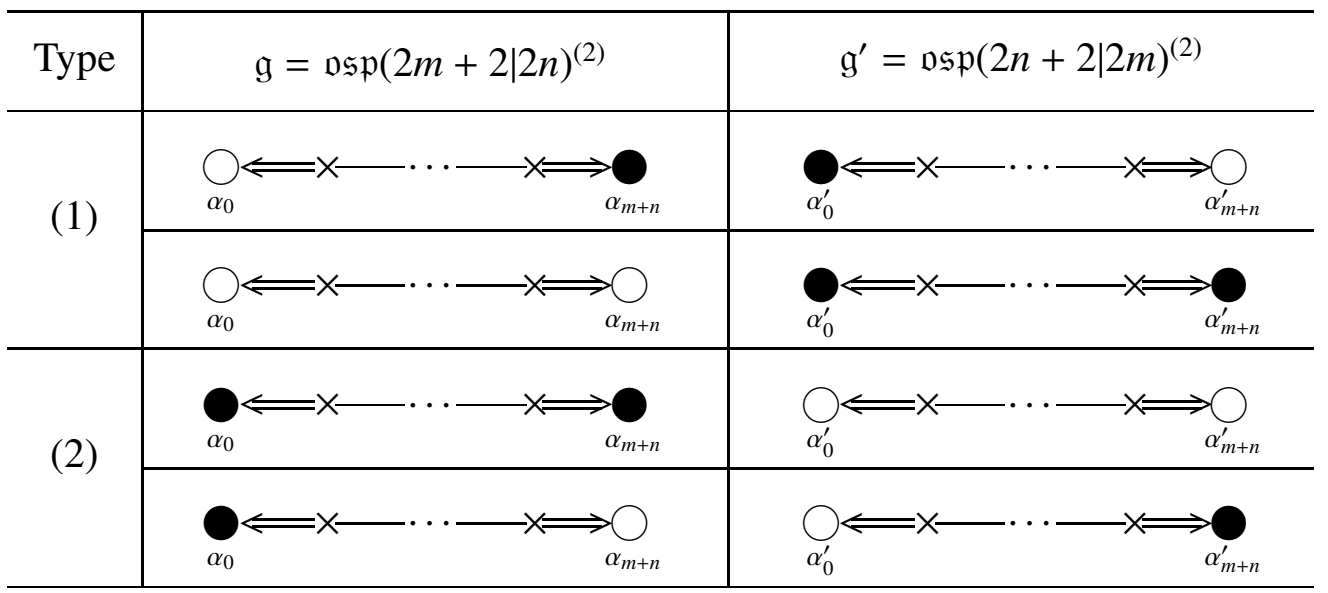

the same as in the previous cases. For $1 \leq i \leq m+n$, the elements $E_{i}, F_{i}, K_{i}, E_{i}^{\prime}, F_{i}^{\prime}, K_{i}^{\prime}$ are given by (3.1); and for $i=0$, they are defined as follows.

Case 1. Type (1) Dynkin diagrams in Table 6.

$$
\begin{aligned}
& E_{0}=\tilde{\Phi}_{2} \prod_{j \in \tau}\left(\tilde{\Phi}_{1} \tilde{\Phi}_{j+1}\right) \cdot e_{0}, \quad F_{0}=\tilde{\Phi}_{1} \prod_{j \in \tau}\left(\tilde{\Phi}_{1} \tilde{\Phi}_{j+1}\right) \cdot f_{0}, \quad K_{0}=\Phi_{1} \cdot k_{0} \\
& E_{0}^{\prime}=\tilde{\Phi}_{2}^{\prime} \prod_{j \in \tau^{\prime}}\left(\tilde{\Phi}_{1}^{\prime} \tilde{\Phi}_{j+1}^{\prime}\right) \cdot e_{0}^{\prime}, \quad F_{0}^{\prime}=\tilde{\Phi}_{1}^{\prime} \prod_{j \in \tau^{\prime}}\left(\tilde{\Phi}_{1}^{\prime} \tilde{\Phi}_{j+1}^{\prime}\right) \cdot f_{0}^{\prime}, \quad K_{0}^{\prime}=\Phi_{1}^{\prime} \cdot k_{0}^{\prime} .
\end{aligned}
$$

In this case, $0 \notin \tau, m+n \in \tau$ while $0 \in \tau^{\prime}, m+n \notin \tau^{\prime}$.

Case 2. Type (2) Dynkin diagrams in Table 6.

$$
\begin{array}{ll}
E_{0}=\Phi_{1} \prod_{j \in \tau}\left(\tilde{\Phi}_{1} \tilde{\Phi}_{j+1}\right) \cdot e_{0}, & F_{0}=\prod_{j \in \tau}\left(\tilde{\Phi}_{1} \tilde{\Phi}_{j+1}\right) \cdot f_{0}, \quad K_{0}=\Phi_{1} \cdot k_{0} ; \\
E_{0}^{\prime}=\tilde{\Phi}_{1}^{\prime} \prod_{j \in \tau^{\prime}}\left(\tilde{\Phi}_{1}^{\prime} \tilde{\Phi}_{j+1}^{\prime}\right) \cdot e_{0}^{\prime}, \quad F_{0}^{\prime}=\prod_{j \in \tau^{\prime}}\left(\tilde{\Phi}_{1}^{\prime} \tilde{\Phi}_{j+1}^{\prime}\right) \cdot f_{0}^{\prime}, \quad K_{0}^{\prime}=\Phi_{1}^{\prime} \cdot k_{0}^{\prime} .
\end{array}
$$

In this case, $0 \in \tau$ and $0 \notin \tau^{\prime}$.

\section{HOPF SUPERALGEBRA ISOMORPHISMS}

We will prove Theorem 1.2 in this section. We begin by discussing some facts on Hopf superalgebras, which are needed presently, but are not expected to be widely known.

\subsection{Picture changes and Drinfeld twists for Hopf superalgebras.}

4.1.1. Picture changes. The category of vector superspaces can be regarded as the category of representations of the group algebra of $\mathbb{Z}_{2}:=\{1, u\}$ where $u^{2}=1$, which is a triangular Hopf algebra with the universal $R$-matrix

$$
R:=\frac{1}{2}(1 \otimes 1+1 \otimes u+u \otimes 1-u \otimes u) \in \mathbb{C}\left[\mathbb{Z}_{2}\right] \otimes \mathbb{C}\left[\mathbb{Z}_{2}\right]
$$


A Hopf superalgebra $\mathscr{H}$ is then a Hopf algebra in this category. The grading of $\mathscr{H}$ is given by the $\mathbb{Z}_{2}$-action such that

$$
\text { u. } a=(-1)^{[a]} a,
$$

for any homogeneous $a \in \mathscr{H}$. For any $a, b \in \mathscr{H}$, if we write their co-products as $\Delta(a)=\sum a_{(1)} \otimes a_{(2)}$ and $\Delta(b)=\sum b_{(1)} \otimes b_{(2)}$ respectively, then $\Delta(a b)$ is given by

$$
\Delta(a b)=(m \otimes m)\left(\sum a_{(1)} \otimes \tau R\left(a_{(2)} \otimes b_{(1)}\right) \otimes b_{(2)}\right)
$$

where $m$ is the multiplication of $\mathscr{H}$, and $\tau: v \otimes w \mapsto w \otimes v$ is the usual permutation map (without signs). Then clearly

$$
\Delta(a b)=\sum(-1)^{\left.\left[b_{(1)}\right)\right]\left[a_{(2)}\right]} a_{(1)} b_{(1)} \otimes a_{(2)} b_{(2)} .
$$

By changing the category of $\mathbb{Z}_{2}$-representations one obtains a non-isomorphic Hopf superalgebra from any given one, such that its category of representations is equivalent to that of the original Hopf superalgebra as tensor category, see [1, Theorem 3.1.1] and [9. Chapter 10.1]. Let us describe this more explicitly.

PC1 ([1, Theorem 3.1.1]). Let $(H, \Delta, \epsilon, S)$ be an ordinary Hopf algebra with a group like element $u$ such that $u^{2}=1$. Using $u$, we decompose $H$ as a vector space into $H=$ $H_{0} \oplus H_{1}$ with

$$
H_{i}=\left\{x \in H \mid u x u^{-1}=(-1)^{i} x\right\} .
$$

This clearly defines a $\mathbb{Z}_{2}$-grading for $H$ as an associative algebra, thus turning it into an superalgebra. We set $[x]=i$ for $x \in H_{i}$. For any $x \in H$, write $\Delta(x)=\Delta_{0}(x)+\Delta_{1}(x)$ with $\Delta_{0}(x) \in H \otimes H_{0}$ and $\Delta_{1}(x) \in H \otimes H_{1}$. Define maps

$$
\begin{array}{ll}
\Delta_{u}: H \longrightarrow H \otimes H, & \Delta_{u}(x)=\Delta_{0}(x)+\Delta_{1}(x)(u \otimes 1), \\
S_{u}: H \longrightarrow H, & S_{u}(x)=u^{[x]} S(x) .
\end{array}
$$

Then $\left(H, \Delta_{u}, \epsilon, S_{u}\right)$ is a Hopf superalgebra. The element $u$ acts as the parity operator (PO) of this Hopf superalgebra in the sense of (4.1).

PC2 ([1, Theorem 3.1.1]). Let $(\mathscr{H}, \Delta, \epsilon, S)$ be a Hopf superalgebra with a group like element $g$ satisfying $g^{2}=1$, which acts as the parity operator in the sense that $g x g^{-1}=$ $(-1)^{[x]} x$ for all homogeneous $x \in \mathscr{H}$. We define maps $\Delta_{g}: \mathscr{H} \longrightarrow \mathscr{H} \otimes \mathscr{H}$ and $S_{g}: \mathscr{H} \longrightarrow \mathscr{H}$ in exactly the same way as in (4.2). Then $\left(\mathscr{H}, \Delta_{g}, \epsilon, S_{g}\right)$ is an ordinary Hopf algebra.

PC. Let $(\mathscr{H}, \Delta, \epsilon, S)$ be a Hopf superalgebra. Suppose that it has two group like elements $g$ and $u$ such that

$$
g^{2}=1=u^{2}, \quad g u=u g, \quad \text { and } g \text { acts as the parity operator. }
$$

We apply PC2 to obtain an ordinary Hopf algebra, and then apply PC1 with $u$ to the ordinary Hopf algebra to obtain a new Hopf superalgebra with parity operator $u$ :

$$
\begin{aligned}
& \text { Hopf superalgebra } \quad{ }_{P C 2} \quad \text { Hopf algebra }{ }_{P C 1} \quad \text { Hopf superalgebra } \\
& (\mathscr{H}, \Delta, \epsilon, S) \stackrel{P C 2}{\sim} \quad\left(\mathscr{H}, \Delta_{g}, \epsilon, S_{g}\right) \stackrel{P C 1}{\sim} \quad\left(\mathscr{H},\left(\Delta_{g}\right)_{u}, \epsilon,\left(S_{g}\right)_{u}\right) \\
& \text { with PO } g \text { with } u \quad \text { with PO } u \text {. }
\end{aligned}
$$

Definition 4.1. Call the operation of constructing the new Hopf superalgebra $\left(\mathscr{H},\left(\Delta_{g}\right)_{u}, \epsilon,\left(S_{g}\right)_{u}\right)$ with parity operator $u$ from a given Hopf superalgebra $(\mathscr{H}, \Delta, \epsilon, S)$ with parity operator $g$ a picture change (PC) with respect to $g$ and $u$. 
Remark 4.2. This is loosely called "bosonisation" in the literature (see [9] in particular). As bosonisation means something very different in quantum field theory, we prefer the term "picture change".

Representation categories of Hopf algebras and Hopf superalgebras are strict tensor categories. For any $\mathscr{H}$-modules $M$ and $N$, the $\mathbb{Z}_{2}$-graded action of $\Delta(x)$ and the ordinary action of $\Delta_{g}(x)$ on $M \otimes N$ coincide for all $x \in \mathscr{H}$. This in essence implies the tensor equivalence of the representation categories of the Hopf superalgebra $(\mathscr{H}, \Delta, \epsilon, S)$ and the ordinary Hopf algebra $\left(\mathscr{H}, \Delta_{g}, \epsilon, S_{g}\right)$ related by PC2. Similarly one can show the tensor equivalence of the representation categories of the Hopf algebra $(H, \Delta, \epsilon, S)$ and Hopf superalgebra $\left(H, \Delta_{u}, \epsilon, S_{u}\right)$ related by PC1. See [1, Theorem 3.1.1].

We summarise the above into the following

Theorem 4.3. Let $(\mathscr{H}, \Delta, \epsilon, S)$ be a Hopf superalgebra with group like elements $g$ and $u$ as described above such that $g$ acts as the parity operator. Then a picture change turns this Hopf superalgebra into a new Hopf superalgebra $\left(\mathscr{H},\left(\Delta_{g}\right)_{u}, \epsilon,\left(S_{g}\right)_{u}\right)$ with parity operator $u$. The categories of representations of the two Hopf superalgebras are equivalent as strict tensor categories.

4.1.2. Twisting the coalgebra structure. Recall the following fact [3, 11]. Let $(\mathscr{H}, \Delta, \epsilon, S)$ be a Hopf superalgebra. Given an invertible even element $\mathcal{J} \in \mathscr{H} \otimes \mathscr{H}$ satisfying the conditions

$$
\begin{aligned}
& (\Delta \otimes \mathrm{id})(\mathcal{J})(\mathcal{J} \otimes 1)=(\mathrm{id} \otimes \Delta)(\mathcal{J})(1 \otimes \mathcal{J}), \\
& (\epsilon \otimes \mathrm{id})(\mathcal{J})=(\mathrm{id} \otimes \epsilon)(\mathcal{J})=1,
\end{aligned}
$$

one can twist the coalgebra structure to obtain a new Hopf superalgebra $\left(\mathscr{H}, \Delta^{\mathcal{J}}, \epsilon, S^{\mathcal{J}}\right)$ with the same underlying associative superalgebraic structure on $\mathscr{H}$. The new comultiplication $\Delta^{\mathcal{J}}$ and antipode $S^{\mathcal{J}}$ are given by

$$
\Delta^{\mathcal{J}}(x)=\mathcal{J}^{-1} \Delta(x) \mathcal{J}, \quad S^{\mathcal{J}}(x)=\mathcal{G}^{-1} S(x) \mathcal{G}, \quad \forall x \in \mathscr{H},
$$

with $\mathcal{G}=m \circ(S \otimes \mathrm{id})(\mathcal{J})$, where $m$ is the multiplication of $\mathscr{H}$. The element $\mathcal{J}$ is called a Drinfeld twist for $\mathscr{H}$. Note that twisting does not change the counit.

4.2. Quantum correspondences. Keep the notation in Section 3, Let $g$ be a Lie superalgebra or affine Lie superalgebra in Table 2 or Table 3 with a fundamental system $\Pi$. Then there exists a corresponding $\mathfrak{g}^{\prime}$ such that $\left(\mathfrak{g}, \mathfrak{g}^{\prime}\right)$ is a pair in Theorem 1.1. Now $\Pi^{\prime}=\phi(\Pi)$ is a fundamental system of $\mathfrak{g}^{\prime}$.

Consider $\mathfrak{U}_{q}(\mathfrak{g}, \Pi)$ as a Hopf superalgebra with the standard grading. As before, we denote its comultiplication, counit and antipode by $\Delta, \epsilon$ and $S$ respectively. Let

$$
u_{1}:=\prod_{i \in \tau}\left(\tilde{\Phi}_{1} \tilde{\Phi}_{i+1}\right), \quad u_{2}:=\tilde{\Phi}_{1} u_{1},
$$

Note that $\tilde{\Phi}_{1} \tilde{\Phi}_{i+1} X_{j}^{ \pm}\left(\tilde{\Phi}_{1} \tilde{\Phi}_{i+1}\right)^{-1}=(-1)^{\delta_{i j}} X_{j}^{ \pm}$for all $i, j$, where $X_{j}^{+}=e_{j}$ and $X_{j}^{-}=f_{j}$. [Recall that $\tilde{\Phi}_{k}=1$ if $k>m+n$ by convention.] Thus $u_{1}$ is the parity operator of $\mathfrak{U}_{q}(\mathfrak{g}, \Pi)$.

Applying a picture change with respect to $u_{1}$ and $u_{2}$ to $\left(\mathfrak{U}_{q}(\mathfrak{g}, \Pi), \Delta, \epsilon, S\right)$, we obtain the Hopf superalgebra $\left(\mathfrak{U}_{q}(\mathfrak{g}, \Pi),\left(\Delta_{u_{1}}\right)_{u_{2}}, \epsilon,\left(S_{u_{1}}\right)_{u_{2}}\right)$ with parity operator $u_{2}$. The new $\mathbb{Z}_{2^{-}}$ grading of $\mathfrak{U}_{q}(\mathfrak{g}, \Pi)$, induced by $u_{2}$, is given by

$$
\begin{aligned}
& \mathfrak{U}_{q}(\mathfrak{g}, \Pi)=\mathfrak{U}_{q}(\mathfrak{g}, \Pi)_{0}^{\prime} \oplus \mathfrak{U}_{q}(\mathfrak{g}, \Pi)_{1}^{\prime} \quad \text { with } \\
& \mathfrak{U}_{q}(\mathfrak{g}, \Pi)_{\theta}^{\prime}=\left\{x \in \mathfrak{U}_{q}(\mathfrak{g}, \Pi) \mid u_{2} x u_{2}^{-1}=(-1)^{\theta} x\right\}, \quad \theta=0,1 .
\end{aligned}
$$


Write $\tilde{\Delta}=\left(\Delta_{u_{1}}\right)_{u_{2}}$ and $\tilde{S}=\left(S_{u_{1}}\right)_{u_{2}}$, and use $\left(\mathfrak{U}_{q}(\mathfrak{g}, \Pi), \tilde{\Delta}, \epsilon, \tilde{S}\right)$ to denote this new Hopf superalgebra with the $\mathbb{Z}_{2}$-grading given by (4.5).

Recall the elements $E_{i}, F_{i}, K_{i}^{ \pm 1}$ of $\mathfrak{U}_{q}(\mathfrak{g}, \Pi)$ introduced in Section 3 , They together with the elements $\sigma_{i}$ generate $\mathfrak{U}_{q}(\mathfrak{g}, \Pi)$. We have the following easy observation.

Lemma 4.4. For any fixed $i$, the elements $E_{i}, F_{i}$ belong to $\mathfrak{U}_{q}(\mathfrak{g}, \Pi)_{0}^{\prime}\left(\right.$ resp. $\left.\mathfrak{U}_{q}(\mathfrak{g}, \Pi)_{1}^{\prime}\right)$ if and only if $\phi\left(\alpha_{i}\right)$ is an even (resp. odd) simple root in $\Pi^{\prime}$.

This immediately implies

Corollary 4.5. The associative algebra isomorphism $\mathfrak{H}_{-q}\left(\mathfrak{g}^{\prime}, \Pi^{\prime}\right) \stackrel{\cong}{\longrightarrow} \mathfrak{U}_{q}(\mathfrak{g}, \Pi)$ of Theorem 1.1 defined by (3.2) is an isomorphism of superalgebras if $\mathfrak{U}_{q}(\mathfrak{g}, \Pi)$ is given the $\mathbb{Z}_{2}$-grading (4.5) induced by $u_{2}$, while $\mathfrak{U}_{-q}\left(\mathfrak{g}^{\prime}, \Pi^{\prime}\right)$ has the usual $\mathbb{Z}_{2}$-grading.

Recall that $|\Pi|$ denotes the cardinality of $\Pi$. Define

$$
\mathcal{J}:=\frac{1}{2^{|\Pi|}} \mathscr{T}, \quad \mathscr{T}:=\mathscr{T}^{(0)} \mathscr{T}^{(1)},
$$

where

$$
\begin{aligned}
& \mathscr{T}^{(0)}:=\prod_{i \notin \tau} \mathscr{T}_{i}^{(0)}, \quad \mathscr{T}^{(1)}:=\prod_{i \in \tau} \mathscr{T}_{i}^{(1)}, \\
& \mathscr{T}_{i}^{(0)}:=\left(1+\tilde{\Phi}_{1} \tilde{\Phi}_{i+1}\right) \otimes 1+\left(1-\tilde{\Phi}_{1} \tilde{\Phi}_{i+1}\right) \otimes \Phi_{i+1}, \quad i \notin \tau, \\
& \mathscr{T}_{i}^{(1)}:=\left(1+\tilde{\Phi}_{1} \tilde{\Phi}_{i+1}\right) \otimes 1+\left(1-\tilde{\Phi}_{1} \tilde{\Phi}_{i+1}\right) \otimes \tilde{\Phi}_{i+2}, \quad i \in \tau .
\end{aligned}
$$

Lemma 4.6. The element $\mathcal{J}$ defined by (4.6) satisfies the relations

$$
\begin{aligned}
& (\tilde{\Delta} \otimes \mathrm{id})(\mathcal{J})(\mathcal{J} \otimes 1)=(\mathrm{id} \otimes \tilde{\Delta})(\mathcal{J})(1 \otimes \mathcal{J}), \\
& (\epsilon \otimes \mathrm{id})(\mathcal{J})=(\mathrm{id} \otimes \epsilon)(\mathcal{J})=1 .
\end{aligned}
$$

Proof. The second relation is clear since $\epsilon\left(\Phi_{i}\right)=\epsilon\left(\tilde{\Phi}_{i}\right)=1$ for all $i$.

To prove the first relation, note that $\mathcal{J}, u_{1}$ and $u_{2}$ involve only the even elements $\sigma_{i}$ of $\mathfrak{U}_{q}(\mathfrak{g}, \Pi)$, which commute among themselves. Thus the first relation is equivalent to that obtained by replacing $\tilde{\Delta}$ by $\Delta$.

For any elements $\sigma, \sigma^{\prime}$ in $\mathrm{G}$, denote $x=(1+\sigma) \otimes 1+(1-\sigma) \otimes \sigma^{\prime}$. It can be proven by direct computations that $(\Delta \otimes \mathrm{id})(x)(x \otimes 1)=(\mathrm{id} \otimes \Delta)(x)(1 \otimes x)$, and hence $(\tilde{\Delta} \otimes \mathrm{id})(x)(x \otimes 1)=$ $(\mathrm{id} \otimes \tilde{\Delta})(x)(1 \otimes x)$. As $\mathscr{T}$ is the product of elements of the form $x$, this immediately leads to the first relation.

Remark 4.7. We have $\mathcal{J}^{-1}=\mathcal{J}$ because $x^{2}=4$ for the $x$ in the proof of the above lemma.

By Lemma4.6, we can twist the Hopf superalgebra $\left(\mathfrak{H}_{q}(\mathfrak{g}, \Pi), \tilde{\Delta}, \epsilon, \tilde{S}\right)$ using the element $\mathcal{J}$ given in (4.6) to obtain a new Hopf superalgebra $\left(\mathfrak{H}_{q}(\mathfrak{g}, \Pi), \tilde{\Delta}^{\mathcal{J}}, \epsilon, \tilde{S}^{\mathcal{J}}\right)$. We emphasize that the $\mathbb{Z}_{2}$-grading is given by (4.5).

Lemma 4.8. The comultiplication, cunit and antipode of the Hopf superalebra $\left(\mathfrak{U}_{q}(\mathfrak{g}, \Pi), \tilde{\Delta}^{\mathcal{J}}, \epsilon, \tilde{S}^{\mathcal{J}}\right)$ are given by

$$
\begin{aligned}
& \tilde{\Delta}^{\mathcal{J}}\left(\sigma_{i}\right)=\sigma_{i} \otimes \sigma_{i}, \quad \tilde{\Delta}^{\mathcal{J}}\left(K_{i}\right)=K_{i} \otimes K_{i}, \\
& \tilde{\Delta}^{\mathcal{J}}\left(E_{i}\right)=E_{i} \otimes 1+K_{i} \otimes E_{i}, \quad \tilde{\Delta}^{\mathcal{J}}\left(F_{i}\right)=F_{i} \otimes K_{i}^{-1}+1 \otimes F_{i}, \\
& \epsilon\left(E_{i}\right)=0, \quad \epsilon\left(F_{i}\right)=0, \quad \epsilon\left(K_{i}\right)=1, \quad \epsilon\left(\sigma_{i}\right)=1 \\
& \tilde{S}^{\mathcal{J}}\left(E_{i}\right)=-K_{i}^{-1} E_{i}, \quad \tilde{S}^{\mathcal{J}}\left(F_{i}\right)=-F_{i} K_{i}, \quad \tilde{S}^{\mathcal{J}}\left(K_{i}\right)=K_{i}^{-1}, \quad \tilde{S}^{\mathcal{J}}\left(\sigma_{i}\right)=\sigma_{i}^{-1}, \quad \forall i .
\end{aligned}
$$


Proof. The relations for the counit are clear, and the antipode relations can be easily obtained from the comultiplication and the counit. Note that $\mathcal{J}$ depends only on $\sigma_{i}$. Since $K_{i}$ and $\sigma_{i}$ are all even and commute among themselves, we immediately have

$$
\tilde{\Delta}^{\mathcal{J}}\left(\sigma_{i}\right)=\tilde{\Delta}\left(\sigma_{i}\right)=\Delta\left(\sigma_{i}\right)=\sigma_{i} \otimes \sigma_{i}, \quad \tilde{\Delta}^{\mathcal{J}}\left(K_{i}\right)=\tilde{\Delta}\left(K_{i}\right)=\Delta\left(K_{i}\right)=K_{i} \otimes K_{i} .
$$

Thus what remains to be proven are the formulae for $\tilde{\Delta}^{\mathcal{J}}\left(E_{i}\right)$ and $\tilde{\Delta}^{\mathcal{T}}\left(F_{i}\right)$. In the Hopf superalgebra $\left(\mathfrak{U}_{q}(\mathfrak{g}, \Pi), \tilde{\Delta}, \varepsilon, \tilde{S}\right)$, we have, for $i, j>0$,

$$
\begin{aligned}
& \tilde{\Delta}\left(E_{i}\right)=E_{i} \otimes \Phi_{i+1}+\Phi_{i} K_{i} \otimes E_{i}, \quad m+n \neq i \notin \tau, \\
& \tilde{\Delta}\left(E_{j}\right)=E_{j} \otimes \tilde{\Phi}_{j+2}+\tilde{\Phi}_{1} \tilde{\Phi}_{j} K_{j} \otimes E_{j}, \quad m+n \neq j \in \tau \\
& \tilde{\Delta}\left(E_{m+n}\right)=E_{m+n} \otimes 1+u \Phi_{m+n} K_{m+n} \otimes E_{m+n},
\end{aligned}
$$

where $u=\tilde{\Phi}_{1} \prod_{j \in \tau, j \neq m+n}\left(\tilde{\Phi}_{1} \tilde{\Phi}_{j+1}\right)$, which is $u_{1}$ if $m+n \in \tau$, and is $u_{2}$ if $m+n \notin \tau$, and

(i) for the Dynkin diagrams of Types (1), (2) and (3) in Table 5

$$
\tilde{\Delta}\left(E_{0}\right)=E_{0} \otimes \Phi_{2}+\Phi_{1} K_{0} \otimes E_{0} ;
$$

(ii) for type (4) Dynkin diagrams in Table 5 ,

$$
\tilde{\Delta}\left(E_{0}\right)=E_{0} \otimes \tilde{\Phi}_{3}+K_{0} \otimes E_{0} ;
$$

(iii) for all Dynkin diagrams in Table 6 ,

$$
\tilde{\Delta}\left(E_{0}\right)=E_{0} \otimes \tilde{\Phi}_{2} \prod_{j \in \tau}\left(\tilde{\Phi}_{1} \tilde{\Phi}_{j+1}\right)+K_{0} \otimes E_{0},
$$

Using the above formulae, we can easily show that

$$
\tilde{\Delta}^{\mathcal{J}}\left(E_{i}\right)=E_{i} \otimes 1+K_{i} \otimes E_{i}, \quad \forall i .
$$

We can similarly prove the formula for $\tilde{\Delta}^{\mathcal{J}}\left(F_{i}\right)$.

Proof of Theorem 1.2 By Corollary 4.5, the map (3.2) is an isomorphism of associative superalgebras, and by Lemma 4.8, it is a Hopf superalgebra map. Hence follows the theorem.

Proof of Theorem 1.4 This easily follows from Theorem 1.2 by using Theorem 4.3

Remark 4.9. We expect that for any pair $\left(\mathfrak{g}, \mathfrak{g}^{\prime}\right)$ in Theorem 1.1, the representation categories of $\mathfrak{U}_{q}(\mathfrak{g}, \Pi)$ and of $\mathfrak{U}_{-q}\left(\mathfrak{g}^{\prime}, \Pi^{\prime}\right)$ are equivalent as braided strict tensor categories. One should be able to prove this following [9, Chapter 10.1].

Remark 4.10. Another possible approach to the proof of Theorem 1.2 is to consider Hopf superalgebras in the category of Yetter-Drinfeld modules. The Hopf superalgebras $\mathfrak{U}_{q}(\mathfrak{g}, \Pi)$ and $\mathfrak{U}_{-q}\left(\mathfrak{g}^{\prime}, \Pi^{\prime}\right)$ are then expected to be quantum doubles of the same Nichols algebra of diagonal type [5]. However, such a proof will necessarily be much more involved.

\section{ACKNOWLEDGEMENTS}

This research was supported by National Natural Science Foundation of China Grants No. 11301130, No. 11431010; and Australian Research Council Discovery-Project Grant DP140103239. Xu wishes to thank the School of Mathematics and Statistics at the University of Sydney for its hospitality. 


\section{REFERENCES}

[1] Andruskiewitsch, N.; Etingof, P.; Gelaki, S., Triangular Hopf algebras with the Chevalley property. Michigan Math. J. 49 (2001), 277-298.

[2] Bracken, A. J.; Gould, M. D.; Zhang, R. B., Quantum supergroups and solutions of the Yang-Baxter equation. Modern Phys. Lett. A. 5 (1990), no. 11, 831-840.

[3] Drinfeld, V. G., Quasi-Hopf algebras. Leningrad Math. J. 1 (1990), no. 6, 1419-1457.

[4] Gorelik, M.; Lanzmann, E., The annihilation theorem for the completely reducible Lie superalgebras. Invent. Math. 137 (1999), no. 3, 651-680.

[5] Heckenberger, I., Lusztig isomorphisms for Drinfel'd doubles of bosonizations of Nichols algebras of diagonal type. J. Alg. 323 (2010), 2130-2180.

[6] Kac, V.G., Lie superalgebras. Adv. Math. 26 (1977), 8-96.

[7] Kac, V.G., Infinite-dimensional Lie algebras. Third edition. Cambridge University Press, Cambridge, 1990.

[8] Lanzmann, E., The $\mathrm{Z}$ transformation and $U_{q}(\operatorname{osp}(1,2 l))$-Verma modules annihilators. Alge. Rep. Theory 5(3) (2002), 235-258.

[9] Majid, S., Foundations of Quantum GroupTheory. Cambridge University Press, 1995.

[10] Mikhaylov, V.; Witten, E., Branes and supergroups. Comm. Math. Phys. 340 (2015), 699-832.

[11] Reshetikhin, N. Multiparameter quantum groups and twisted quasitriangular Hopf algebras. Lett. Math. Phys. 20 (1990), no. 4, 331-335.

[12] Rittenberg, V.; Scheunert, M., A remarkable connection between the representations of the Lie superalgebras $\mathfrak{d} \mathfrak{s p}(1,2 n)$ and the Lie algebras $o(2 n+1)$. Comm. Math. Phys. 83 (1982), no. 1, 1-9.

[13] van de Leur, J.W., Contragredient Lie superalgebras of finite growth. Utrecht thesis (1985).

[14] Xu, Y,; Zhang, R. B. Drinfeld realisations of quantum affine superalgebras. arXiv:1611.06449.

[15] Yamane, H., Universal $R$-matrices for quantum groups associated to simple Lie superalgebras. Proc. Japan Academy, Series A, Math Sciences 67(4) (1991), 108-112.

[16] Yamane, H., On defining relations of affine Lie superalgebras and affine quantized universal enveloping superalgebras. Publ. Res. Inst. Math. Sci. 35 (1999), no. 3, 321-390.

[17] Zhang, R. B., Finite-dimensional representations of $\mathrm{U}_{q}(\operatorname{osp}(1 / 2 n))$ and its connection with quantum so $(2 n+1)$. Lett. Math. Physics, 25 (1992), 317-325.

[18] Zhang, R. B., Finite dimensional irreducible representations of the quantum supergroup $\mathrm{U}_{\mathrm{q}}(g l(m / n))$. J. Math Phys. 34 (1993), 1236-1254.

[19] Zhang, R. B., Symmetrizable quantum affine superalgebras and their representations. J. Math Phys. 38 (1997), 535-543.

[20] Zhang, R. B., Structure and representations of the quantum general linear supergroup. Comm. Math. Phys. 195 (1998), no. 3, 525-547.

[21] Zhang, R.B., Serre presentations of Lie superalgebras. In Advances in Lie Superalgebras, 235-280, Springer INdAM Ser., 7, Springer, Cham, 2014.

[22] Zhang, R. B., M D Gould and A J Bracken, Solutions of the graded classical Yang-Baxter equation and integrable models. J. Phys. A: Math. Gen. 24 (1991), 1185-1197.

(Xu) School of Mathematics, Hefei University of Technology, Anhui Province, 230009, China

(Xu, Zhang) School of Mathematics and Statistics, University of Sydney, NSW 2006, Australia

E-mail address: yingxu@maths.usyd.edu.au, ruibin.zhang@sydney.edu.au 